\title{
The $130-370 \mathrm{GHz}$ rotational spectrum of phenyl isocyanide $\left(\mathrm{C}_{6} \mathrm{H}_{5} \mathrm{NC}\right)$
}

\author{
Cite as: J. Chem. Phys. 151, 024301 (2019); doi: 10.1063/1.5100805 \\ Submitted: 20 April 2019 - Accepted: 7 June 2019 • \\ Published Online: 8 July 2019
}

Maria A. Zdanovskaia, (D) Brian J. Esselman, (D) R. Claude Woods, and Robert J. McMahon ${ }^{\text {a) }}$

AFFILIATIONS
Department of Chemistry, University of Wisconsin-Madison, Madison, Wisconsin 53706, USA

a) Authors to whom correspondence should be addressed: rcwoods@wisc.edu and robert.mcmahon@wisc.edu

\begin{abstract}
The analysis of phenyl isocyanide $\left(\mathrm{C}_{6} \mathrm{H}_{5} \mathrm{NC}, \mu_{a}=4.0 \mathrm{D}\right)$ in its ground vibrational state and two lowest-energy excited vibrational states, $v_{22}$ $\left(141 \mathrm{~cm}^{-1}\right)$ and $v_{33}\left(155 \mathrm{~cm}^{-1}\right)$, in the $130-370 \mathrm{GHz}$ frequency region has been completed. Over 4500 new rotational transitions have been measured in the ground vibrational state for the most abundant isotopologue, resulting in the determination of the spectroscopic constants for a partial octic Hamiltonian with low error. The Coriolis-coupled $v_{22}-v_{33}$ dyad reported herein, containing over 3500 new transitions for each vibrational state, has been analyzed for the first time. The coupled-state least-squares fit utilizes seven coupling terms $\left(G_{a}, G_{a}^{J}, G_{a}^{K}, G_{a}^{J J}\right.$, $G_{a}^{J K}, F_{b c}$, and $\left.F_{b c}^{K}\right)$ to address perturbation between the two vibrational states, including resonances and several nominal interstate transitions. This work results in precise determination of the energy separation between the two states, $\Delta E_{22,33}=9.682248(3) \mathrm{cm}^{-1}$, and the Coriolis coupling coefficient, $\left|\zeta_{22,33}^{a}\right|=0.858(9)$. The precise rotational and distortion constants determined in this work provide the foundation for an astronomical search for phenyl isocyanide across the radio band.
\end{abstract}

Published under license by AIP Publishing. https://doi.org/10.1063/1.5100805

\section{INTRODUCTION}

The recent detection of benzonitrile $\left(\mathrm{C}_{6} \mathrm{H}_{5} \mathrm{CN}\right)^{1}$ in Taurus Molecular Cloud 1 (TMC-1 $)^{2}$-the first detection of a simple derivative of benzene in the interstellar medium (ISM) by radioastronomy-invites consideration of the isomeric molecule, phenyl isocyanide $\left(\mathrm{C}_{6} \mathrm{H}_{5} \mathrm{NC}\right)$. In the current study, we describe a detailed analysis of the rotational spectrum of phenyl isocyanide in its ground vibrational state and two lowest-energy excited vibrational states, $v_{22}\left(141 \mathrm{~cm}^{-1}\right)$ and $v_{33}\left(155 \mathrm{~cm}^{-1}\right)$, in the $130-375 \mathrm{GHz}$ frequency region. Phenyl isocyanide $\left(\mathrm{C}_{6} \mathrm{H}_{5} \mathrm{NC}\right.$, Fig. 1) is a planar, prolate $(\kappa=-0.832),{ }^{3}$ asymmetric top rotor with its dipole moment along the $a$-inertial axis $\left(\mu_{a}=4.0 \mathrm{D}\right),{ }^{4}$ resulting in a spectrum dominated by ${ }^{a} \mathrm{R}$-type absorptions. Its infrared, ${ }^{5}$ Raman, and $12-40 \mathrm{GHz}$ microwave ${ }^{3}$ spectra have been studied, the latter leading to a microwave substitution structure based upon measurement of four isotopologues $\left(\mathrm{C}_{6} \mathrm{H}_{5} \mathrm{NC}, 2-\left[{ }^{2} \mathrm{H}\right]-\mathrm{C}_{6} \mathrm{H}_{5} \mathrm{NC}, 2,4-\right.$ $\left.\left[{ }^{2} \mathrm{H}\right]-\mathrm{C}_{6} \mathrm{H}_{5} \mathrm{NC}, 2,4,6-\left[{ }^{2} \mathrm{H}\right]-\mathrm{C}_{6} \mathrm{H}_{5} \mathrm{NC}\right)$. Kasten et al. later analyzed the ${ }^{14} \mathrm{~N}$ nuclear quadrupole hyperfine splitting using transitions in the 5-8 GHz frequency range. No previous studies, however, examined the rotational spectrum of phenyl isocyanide at frequencies higher than $40 \mathrm{GHz}$. The precise determination of its rotational and distortion constants using an extended frequency range provides the information necessary for an astronomical search for phenyl isocyanide in the ISM by radioastronomy. Its identification and measurement of abundance relative to its nitrile counterpart could help inform our understanding of interstellar chemistry.

Detecting aromatic molecules in the ISM by radioastronomy and understanding the role of aromatic compounds in interstellar chemistry are goals of longstanding interest in astrochemistry. ${ }^{6-}$ The small, $2 \pi$-electron aromatic compounds cyclopropenylidene ${ }^{9,1}$ and cyclopropenone ${ }^{11}$ have been detected by radioastronomy, and larger aromatic compounds (benzene, $\mathrm{C}_{60}, \mathrm{C}_{70}$ ) have been detected by infrared spectroscopy. ${ }^{12,13}$ Benzonitrile has long been an alluring target for radioastronomers due to its strong dipole moment $\left(\mu_{a}=4.5 \mathrm{D}\right)^{14}$ and the fact that many nitriles have been detected in the ISM. ${ }^{15}$ It has been suggested as a tracer molecule for benzene in the ISM and, as previously mentioned, was recently detected by radioastronomy ${ }^{1}$ in TMC-1.

Several nitriles and their corresponding isocyanides have been observed in the ISM: the SiCN and SiNC radicals, ${ }^{16,17}$ the $\mathrm{MgCN}$ and $\mathrm{MgNC}$ radicals, ${ }^{18-20}$ cyanogen (inferred from the observation 


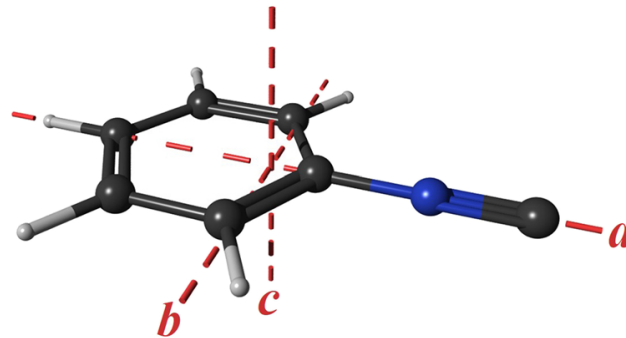

FIG. 1. Phenyl isocyanide structure with principal rotation axes.

of its protonated form $)^{21}$ and isocyanogen, ${ }^{22}$ cyanoacetylene and isocyanoacetylene, ${ }^{23-27}$ hydrogen cyanide and hydrogen isocyanide, ${ }^{27-31}$ their deuterated counterparts, ${ }^{32}$ and methyl cyanide and methyl isocyanide. ${ }^{3-35}$ In some cases where both isomers are detected, their relative abundances have been estimated. Cernicharo et al. detected $\mathrm{CH}_{3} \mathrm{NC}$ toward Sagittarius B2 (Sgr B2) and approximated its abundance relative to $\mathrm{CH}_{3} \mathrm{CN}$ to be $0.05: 1{ }^{33}$ Remijan et al. later estimated this ratio to be $0.02: 1$ and suggested that the isocyanide is formed by nonthermal processes. ${ }^{34}$ Gratier et al. approximated the two species to be in a $0.15: 1$ ratio in the photodissociation region of the Horsehead mane. ${ }^{35}$ In general, an organic isocyanide $(\mathrm{R}-\mathrm{N} \equiv \mathrm{C}$ ) is $\sim 21 \mathrm{kcal} / \mathrm{mol}$ higher in energy than the corresponding nitrile $(\mathrm{R}-\mathrm{C} \equiv \mathrm{N})$, and this energy difference is remarkably insensitive to the nature of the substituent (R). ${ }^{36}$ Hudson and Moore conducted proton bombardment and UV photolysis experiments on ices containing nitriles and demonstrated that both treatments, in the absence of water, resulted in the generation of the corresponding isocyanides. ${ }^{37}$ It is plausible that phenyl isocyanide may be detectable in the interstellar medium, particularly in regions whose chemistry is governed by pulse shocks, irradiation, or other nonthermal processes.

In the current study, we collected the rotational spectrum of phenyl isocyanide in the $130-370 \mathrm{GHz}$ frequency region. The ground vibrational state was fit to a single-state distorted rotor model using over 4500 transitions. The two lowest-energy excited vibrational states, $v_{22}\left(141 \mathrm{~cm}^{-1}, B_{1}\right.$ symmetry $)$ and $v_{33}\left(155 \mathrm{~cm}^{-1}\right.$, $B_{2}$ symmetry), are reported for the first time. They exhibit strong Coriolis coupling and are least-squares fit to a two-state model. This model addresses resonances and allows the measurement of several nominal interstate transitions, resulting in a highly precise determination of their energy separation. An unperturbed ground vibrational state separated by approximately $150 \mathrm{~cm}^{-1}$ from a dyad of low-lying, coupled vibrational states is observed for similar molecules, e.g., phenylacetylene ${ }^{38}$ and benzonitrile. ${ }^{39}$

\section{EXPERIMENTAL METHODS}

A sample of phenyl isocyanide was prepared as described later. Spectra were collected on a broadband spectrometer using 5-10 mTorr sample pressure at room temperature. The 250-370 GHz spectrum was collected using the instrument described previously. ${ }^{39-41}$ The $130-230 \mathrm{GHz}$ frequency spectrum was collected on the same apparatus using a Virginia Diodes amplification and multiplication chain (WR5.1AMC 140-220 GHz) and a Virginia Diodes zero-bias detector (WR5.1ZBD 140-220 GHz). The spectra were combined into a single broadband spectrum using Assignment and Analysis of Broadband Spectra (AABS) software. ${ }^{42,43}$ Pickett's SPFIT/SPCAT programs were used to conduct least-squares fitting and spectral prediction, respectively. ${ }^{44}$ PIFORM, PMIXC, PLANM, and AC programs were used for data analysis, to reformat output files, and to generate various plots.

In our least-squares fits, we assume a uniform frequency measurement uncertainty of $50 \mathrm{kHz}$ for our own data, as well as for the data reported by Kasten et al. ${ }^{4}$ (Uncertainties were not provided in the original publication.)

\section{Computational Methods}

All computations were performed at the B3LYP/6-311+G(2d,p) level of theory using Gaussian 16. ${ }^{46}$ An optimized geometry for phenyl isocyanide was obtained using very tight convergence criteria and an ultrafine integration grid. Anharmonic vibrational frequency calculations provided the fundamental vibrations and the vibrationrotation interaction constants $\left(\alpha_{i}\right)$. For comparison to the observable rotational constants, $A_{0}, B_{0}$, and $C_{0}$, the $A_{e}, B_{e}$, and $C_{e}$ equilibrium rotational constants obtained from the B3LYP calculation were corrected by one half the sum of the computed vibration-rotation interaction constants. Optimized geometry and anharmonic vibrational frequency calculations for benzonitrile, using the same criteria and level of theory, were obtained for comparison. Computational output files are provided in the supplementary material. Tables of the vibration-rotation interaction constants for the two isomers are also provided in the supplementary material.

\section{Synthesis}

Phenyl isocyanide was prepared using a procedure adapted from the work of Leifert et al. ${ }^{47}$ (Scheme 1). In a 1-L round-bottom flask under nitrogen and cooled in an ice/acetone bath $\left(-10{ }^{\circ} \mathrm{C}\right)$, $15 \mathrm{~g}$ formanilide (124 mmol, 1 equivalent) was dissolved in $200 \mathrm{ml}$ tetrahydrofuran (THF). To the solution was added $103.6 \mathrm{ml}$ triethylamine (743 mmol, 6 equivalents). Subsequently, $17.3 \mathrm{ml}$ phosphoryl trichloride $\left(\mathrm{POCl}_{3} ; 185 \mathrm{mmol}, 1.5\right.$ equivalents) was added dropwise to avoid vigorous reaction. The solution changed from clear, light yellow to orange and opaque to dark brown over the course of the addition. The acetone/ice-cooled reaction was stirred for $2 \mathrm{~h}$. A $10 \%$ aqueous solution of $\mathrm{Na}_{2} \mathrm{CO}_{3}$ was added slowly with stirring $(200 \mathrm{ml})$. The mixture was then warmed to room temperature and allowed to stir for $1 \mathrm{~h}$. The organic layer was separated, and the aqueous layer was extracted with dichloromethane. The combined organic layers were dried over $\mathrm{MgSO}_{4}$, filtered, and concentrated by rotary evaporation. Phenyl isocyanide (bp $52-53{ }^{\circ} \mathrm{C}$ at 12 Torr $)^{48}$ was purified by vacuum distillation $\left(\sim 50{ }^{\circ} \mathrm{C}\right)$ and collected

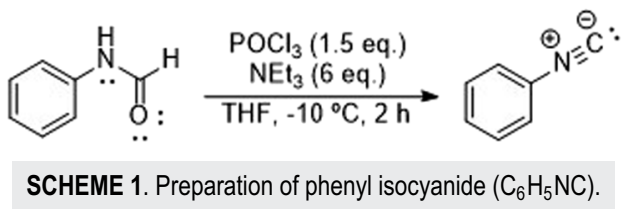


in a receiving flask cooled with a dry-ice/acetone bath. The ${ }^{1} \mathrm{H}$ and ${ }^{13} \mathrm{C}-\mathrm{NMR}$ spectra appeared as expected based on literature data ${ }^{49,50}$ and are provided in the supplementary material, along with a mass spectrum.

As is characteristic of many isocyanides, ${ }^{51}$ phenyl isocyanide has a very strong, repulsive, pungent odor and should be treated with caution. All materials that came in contact with phenyl isocyanide were promptly and thoroughly rinsed with $5 \%$ sulfuric acid in methanol to remove the stench and then washed.

\section{ANALYSIS OF ROTATIONAL SPECTRA}

\section{Ground state}

The phenyl isocyanide spectrum, like that of its nitrile isomer, exhibits numerous vibrational states in the $130-370 \mathrm{GHz}$ region, resulting in a high spectral line density. A portion of the experimental spectrum in the $166220-167200 \mathrm{MHz}$ region is provided in Fig. 2, which highlights the ground vibrational state and its two lowest-lying fundamentals, $v_{22}$ and $v_{33}$. The phenyl isocyanide spectrum is predominantly composed of ${ }^{a} \mathrm{R}_{0,1}$ bands (separated by approximately $2500 \mathrm{MHz}, \sim 2 C$ ); no P- or Q-branch transitions were observed in this work due to their very low intensity in the frequency range examined. The ${ }^{a} \mathrm{R}_{0,1}$ sub-bands begin with $K_{a}=0$ at low frequency and spread out to higher frequency with increasing $K_{a}$ and concurrently decreasing $J$. In the observed region, the lead line is composed of two degenerate transitions that have equal values of $K_{c}$. As a result, the two degenerate transitions have quantum numbers $K_{a}+K_{c}=J$ and $K_{a}+K_{c}=J+1$, referred to below using a + or - superscript on the value of $K_{a}$, respectively, for brevity. With increasing $K_{a}$, the transitions lose degeneracy after which point the band undergoes a turnaround. At even greater values of $K_{a}$, pairs of + and - symmetry lines become degenerate with equal values of $K_{a}$. Many of these $K_{a}$-degenerate ${ }^{a} \mathrm{R}_{0,1}$ transitions are observable in the lower frequency range, $130-220 \mathrm{GHz}$. The higher frequency bands become more compact, and the loss of degeneracy and turnaround occur at higher $K_{a}$. The loss of degeneracy occurs at $J^{\prime \prime}+1=59$ in the ground state band depicted in Fig. 2. The turnaround for this band occurs after $K_{a}=10\left(J^{\prime \prime}+1=55\right)$.

The vibrational ground state of phenyl isocyanide has been least-squares fit to a single-state distorted rotor model including ${ }^{14} \mathrm{~N}$ hyperfine-resolved transitions from the work of Kasten et al. ${ }^{4}$ The transitions of Bak et $a .^{3}$ were not available and thus not included in this work. We have measured over 4500 new lines; $J^{\prime \prime}$ ranges from 42 to 144 and $K_{a}$ ranges from 0 to 66 . The resulting spectroscopic constants $\left(A \text {-reduction, } I^{\mathrm{r}} \text { representation }\right)^{52}$ are presented in Table I, along with those of Bak et al. ${ }^{3}$ and Kasten et al., ${ }^{4}$ those predicted by an anharmonic frequency calculation at the B3LYP/6$311+\mathrm{G}(2 \mathrm{~d}, \mathrm{p})$ level of theory, and the spectroscopic constants of benzonitrile $^{39}$ for comparison. A data distribution plot of the ground vibrational state is presented in Fig. 3, which shows the scope of data collected. In such a plot, the size of the circle corresponds to the magnitude of the error for the line measurement. The plot in Fig. 3 shows that there are lines with somewhat high error scattered throughout the data set, suggesting that there may be underlying features or other nonsystematic error in the spectrum collected. The

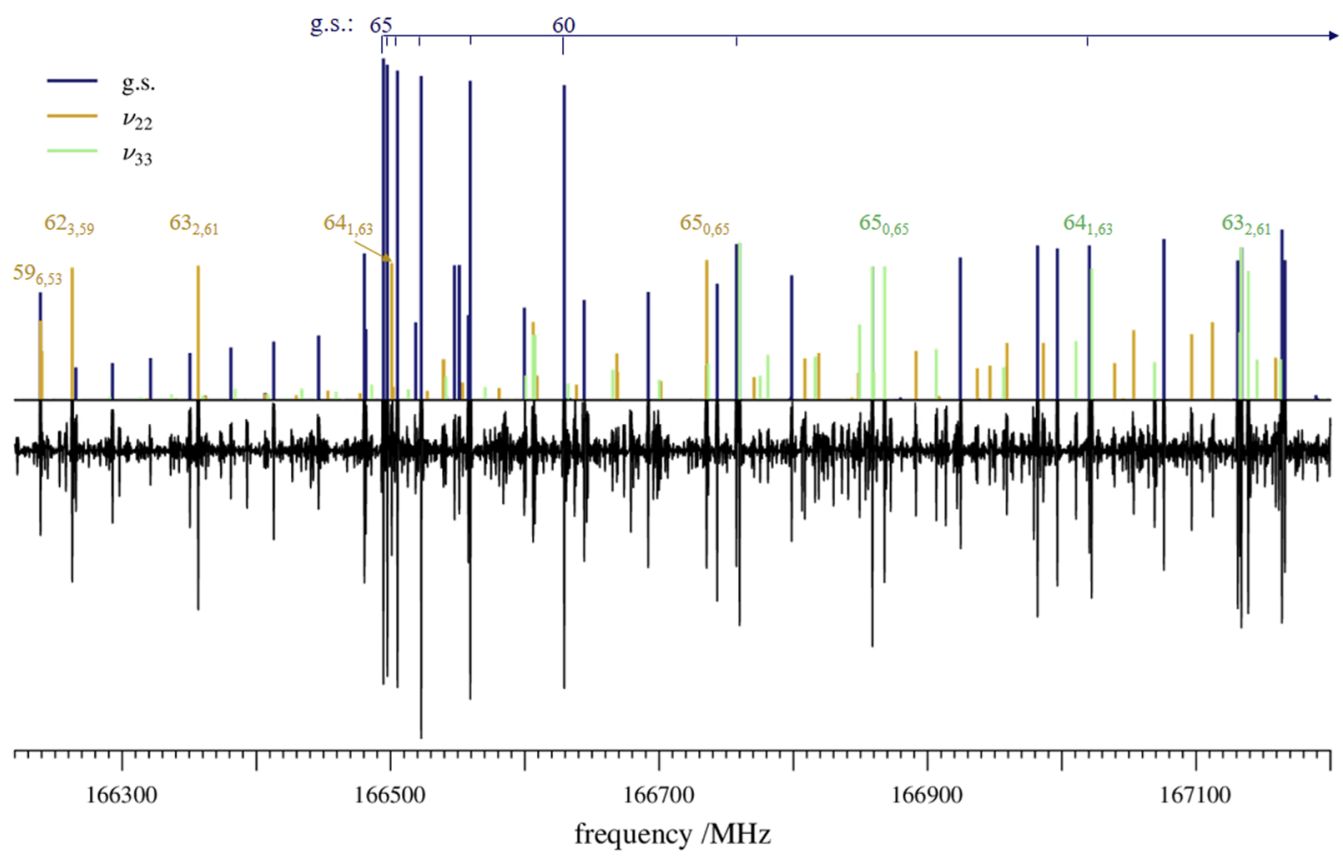

FIG. 2. Phenyl isocyanide rotational spectrum from $166220 \mathrm{MHz}$ to $167200 \mathrm{MHz}$ (bottom) and stick spectra of the ground vibrational state, $v_{22}$, and $v_{33}$ transitions (top). The upper state $J$ (i.e., $J^{\prime \prime}+1$ ) values are marked for ground state lines (blue), whereas all three upper rotational state quantum numbers are marked for $v_{22}$ (gold) and $v_{33}$ (green). Many transitions belonging to other vibrational satellites are also visible in the spectrum. 
TABLE I. Experimental and computational spectroscopic constants for the ground vibrational state of phenyl isocyanide $\left(\mathrm{C}_{6} \mathrm{H}_{5} \mathrm{NC}\right)$ and experimental spectroscopic constants for the ground vibrational state of benzonitrile $\left(\mathrm{C}_{6} \mathrm{H}_{5} \mathrm{CN}\right)$.

\begin{tabular}{|c|c|c|c|c|}
\hline & Bak et $a ._{.}^{3}$ and Kasten et al. ${ }^{4, \mathrm{a}, \mathrm{b}}$ & Current work $^{c}$ & B3LYP $^{\mathrm{d}}$ & Benzonitrile $^{39}$ \\
\hline$A_{0}(\mathrm{MHz})$ & $5659.5190(56)$ & $5659.3374(13)$ & 5658.4 & $5655.265371(75)$ \\
\hline$B_{0}(\mathrm{MHz})$ & $1639.7757(12)$ & $1639.771667(59)$ & 1635.8 & $1546.8757804(76)$ \\
\hline$C_{0}(\mathrm{MHz})$ & $1271.1538(12)$ & $1271.154548(49)$ & 1268.7 & $1214.4040772(67)$ \\
\hline$\Delta_{J}(\mathrm{kHz})$ & $0.0473(27)$ & $0.0494489(40)$ & 0.0472 & $0.0452653(27)$ \\
\hline$\Delta_{J K}(\mathrm{kHz})$ & $0.853(12)$ & $0.912873(36)$ & 0.878 & $0.937906(27)$ \\
\hline$\Delta_{K}(\mathrm{kHz})$ & & $0.2605(17)$ & 0.265 & $0.24234(77)$ \\
\hline$\delta_{J}(\mathrm{kHz})$ & $0.0187(14)$ & $0.0124650(22)$ & 0.0118 & $0.01101589(73)$ \\
\hline$\delta_{K}(\mathrm{kHz})$ & & $0.60134(13)$ & 0.572 & $0.609088(74)$ \\
\hline$\Phi_{J}(\mathrm{~Hz})$ & & $0.00000262(17)$ & 0.00000252 & $0.00000051(22)$ \\
\hline$\Phi_{J K}(\mathrm{~Hz})$ & & $0.0018134(85)$ & 0.00167 & $0.0015435(46)$ \\
\hline$\Phi_{K J}(\mathrm{~Hz})$ & & $-0.009494(31)$ & -0.00868 & $-0.007849(17)$ \\
\hline$\Phi_{K}(\mathrm{~Hz})$ & & {$[0.00755]$} & 0.00756 & {$[0]$} \\
\hline$\phi_{J}(\mathrm{~Hz})$ & & $0.000001206(86)$ & 0.00000118 & $0.000001412(38)$ \\
\hline$\phi_{J K}(\mathrm{~Hz})$ & & $0.0008555(58)$ & 0.000842 & $0.0007431(30)$ \\
\hline$\phi_{K}(\mathrm{~Hz})$ & & $0.00651(13)$ & 0.00641 & $0.007106(76)$ \\
\hline$L_{J}(\mu \mathrm{Hz})$ & & {$[0]$.} & & $0.0000585(56)$ \\
\hline$L_{J J K}(\mu \mathrm{Hz})$ & & $-0.003943(94)$ & & $-0.002085(52)$ \\
\hline$L_{J K}(\mu \mathrm{Hz})$ & & {$[0]$.} & & \\
\hline$L_{K K J}(\mu \mathrm{Hz})$ & & $-0.0327(35)$ & & $-0.0428(18)$ \\
\hline$L_{K}(\mu \mathrm{Hz})$ & & $6.38(15)$ & & $4.468(90)$ \\
\hline$\Delta_{i}\left(\mathrm{u} \AA^{2}\right)^{\mathrm{e}}$ & & $0.073921(28)$ & $0.07996^{\mathrm{f}}$ & $0.080084(3)$ \\
\hline$N^{g}$ & & 4723 & & 4073 \\
\hline$\sigma_{\text {fit }}(\mathrm{MHz})$ & 0.004 & 0.034 & & 0.025 \\
\hline
\end{tabular}

${ }^{a}$ Kasten et al. ${ }^{4}$ included the rotational constants of Bak et al.

${ }^{\mathrm{b}}$ Distortion constants reported as $T_{4}$ and $T_{5}$ converted to A-reduction distortion constants.

${ }^{\mathrm{c}}$ Global fit to the present millimeter-wave measurements and the available literature data.

${ }^{\mathrm{d}}$ Evaluated with the $6-311+\mathrm{G}(2 \mathrm{~d}, \mathrm{p})$ basis set and with constants converted to a right-handed coordinate system by reversing the signs of all off-diagonal (lowercase) distortion constants.

${ }^{\mathrm{e}}$ Inertial defect, $\Delta_{i}=I_{c}-I_{a}-I_{b}$.

${ }^{\mathrm{f}}$ Calculated from the $B_{0}$ constants using the PLANM program.

${ }^{\mathrm{g}}$ Number of fitted transition frequencies.

fact that a large proportion of such lines occurs at high $K_{a}$ and $J^{\prime \prime}$ may suggest an unaddressed systematic issue, although attempts to incorporate additional spectroscopic constants into the least-squares fit were unsuccessful in improving the error.

Despite a lack of Q-branch transitions, the very large data set enabled us to determine several purely $K$-dependent terms: $\Delta_{K}, \delta_{K}$, $\phi_{K}$, and $L_{K}$. The precision of all of the determined constants except $A_{0}$ have been improved by at least two orders of magnitude compared to those previously determined. ${ }^{3}$ The older constants are reasonably confirmed by those determined in the current work. The previously determined values of $A_{0}, \Delta_{J K}$, and $\delta_{J K}$, however, do differ from the more precisely determined constants by somewhat more than their quoted error limits. We have newly determined $\Delta_{K}$, all of the sextic centrifugal distortion constants except $\Phi_{K}$ and three of the on-diagonal octic centrifugal distortion terms $\left(L_{J J K}, L_{K K J}\right.$, and $\left.L_{K}\right)$. Unlike in the case of benzonitrile, ${ }^{53}$ however, we were unable to improve upon the precision of the quadrupole coupling constants for phenyl isocyanide. The experimentally determined constants are also in excellent agreement with those predicted by the anharmonic calculation at the B3LYP/6-311+G(2d,p) level of theory. The relative errors of the B3LYP predictions are $0.02 \%$ for $A_{0}$, $0.2 \%$ for $B_{0}$, and $0.2 \%$ for $C_{0}$. We were unable to determine $\Phi_{K}$, which we fixed to the B3LYP-predicted value, but the quartic and other sextic centrifugal distortion constants reported in this work show remarkable agreement (to within 10\%) with those predicted computationally.

In comparing benzonitrile and phenyl isocyanide, the $A_{0}$ rotational constants are quite similar. That of phenyl isocyanide is approximately $4 \mathrm{MHz}$ greater than that of benzonitrile. Interestingly, the values of one half the sum of the vibration-rotation interaction constants for the two isomers are nearly equal $(41.856 \mathrm{MHz}$ for phenyl isocyanide and $41.992 \mathrm{MHz}$ for benzonitrile). Since this 


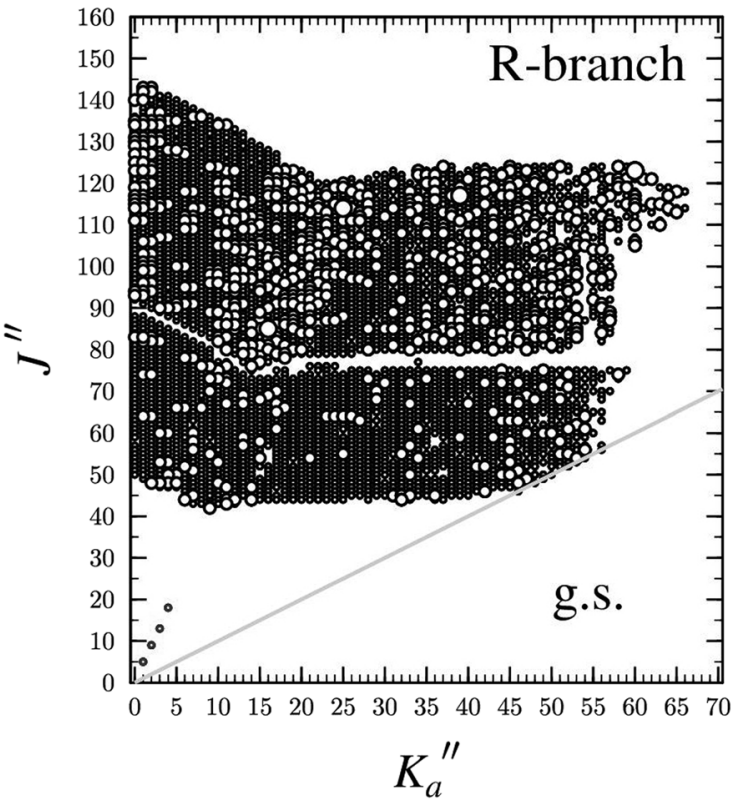

FIG. 3. Data distribution plot for the least-squares fit of spectroscopic data from the current work (black circles) and measurements from the work of Kasten et al. ${ }^{4}$ (blue circles) for the vibrational ground state of phenyl isocyanide. The size of the plotted symbol is proportional to the value of $\left|\left(f_{\text {obs. }}-f_{\text {calc. }}\right) / \delta f\right|$, where $\delta f$ is the frequency measurement uncertainty $(50 \mathrm{kHz})$, and all values shown are smaller than 3.

value is expected to be accurate from these B3LYP computational predictions, ${ }^{54,55}$ the observed difference between the $A_{0}$ rotational constants appears to be indicative of a slight contraction of the phenyl isocyanide aromatic ring toward the $a$ principal axis relative to that of benzonitrile (corresponding to $<0.0004 \AA$ in the $b$ coordinate of each off- $a$-axis $C$ atom). The $B_{0}$ and $C_{0}$ rotational constants, on the other hand, are markedly different between the two isomers ( $93 \mathrm{MHz}$ and $\sim 57 \mathrm{MHz}$, respectively, greater for phenyl isocyanide than for benzonitrile). The difference arises because the $\mathrm{C}$ and $\mathrm{N}$ atoms whose positions differ are located off-axis relative to the $b$ and $c$ axes. The quartic centrifugal distortion constants are also highly similar; all those of phenyl isocyanide (except $\delta_{J}$ ) are within $10 \%$ of those for benzonitrile. The purely $K$-dependent terms are most similar, whereas the purely $J$-dependent terms are most different. For both isomers, $\Phi_{K}$ was the only undetermined sextic centrifugal distortion constant. Of those that were determined, the $\Phi_{J}$ terms differ by an order of magnitude, while the other sextic constants are within $20 \%$ of one another. The three octic centrifugal distortion terms that were determined for both molecules are within $50 \%$ of one another. Altogether, the close agreement between the distortion constants supports the idea that the values are physically meaningful for both isomers.

\section{The lowest-energy vibrational states $v_{22}$ and $v_{33}$}

Phenyl isocyanide exhibits a Coriolis-coupled dyad of lowlying excited vibrational states, similar to the vibrational manifold of benzonitrile. The lower energy fundamental, $v_{22}\left(141 \mathrm{~cm}^{-1}, B_{1} \mathrm{sym}-\right.$ metry), is the out-of-plane bend of the isocyanide group $(\mathrm{R}-\mathrm{N} \equiv \mathrm{C})$ relative to the aromatic ring and $v_{33}\left(155 \mathrm{~cm}^{-1}, B_{2}\right.$ symmetry) is the bend of the isocyanide in the plane of the ring. The vibrational energy levels are depicted in Fig. 4. A tetrad of states lies approximately $300 \mathrm{~cm}^{-1}$ above the ground vibrational state. The tetrad includes the first overtone and combination states of $v_{22}$ and $v_{33}$, as well as the fundamental state $v_{21}$. Higher in energy still, between 400 and $500 \mathrm{~cm}^{-1}$ above the ground vibrational state, lies a complex polyad of overtone, combination, and additional fundamental states. A table of all fundamental modes, their symmetries, harmonic frequencies, anharmonic frequencies, and corresponding infrared intensities at the B3LYP/6-311+G(2d,p) level of theory is provided in the supplementary material.

Like the ground vibrational state, bands of ${ }^{a} \mathrm{R}$ transitions dominate the millimeter-wave spectra of the vibrational states. In our spectral region, both of the lowest-energy vibrational states follow the same degeneracy pattern as the ground state, where lines with low $K_{a}{ }^{+}$are degenerate with the $K_{a}{ }^{-}$transition with the same value of $K_{c}$. They lose this degeneracy with increasing $K_{a}$ and eventually become degenerate $K_{a}{ }^{+}, K_{a}{ }^{-}$pairs with matching values of $K_{a}$. In $v_{22}$, bands initially progress toward lower frequency moving away from the lead transition. The band undergoes two turnarounds and eventually progresses to lower frequency with increasing $K_{a}$ and concurrently decreasing $J^{\prime \prime}$. In the band depicted in Fig. 2 , the first turnaround occurs after $J^{\prime \prime}+1=60$ and the initial loss of degeneracy occurs at $J^{\prime \prime}+1=59$.

In the lower-frequency portion of our spectral region, $v_{33}$ behaves like the ground state. Moving away from the bandhead, transitions within a band proceed toward higher frequency and then turn around and progress to lower frequency. This is the case for the band depicted in Fig. 2, although the loss of degeneracy

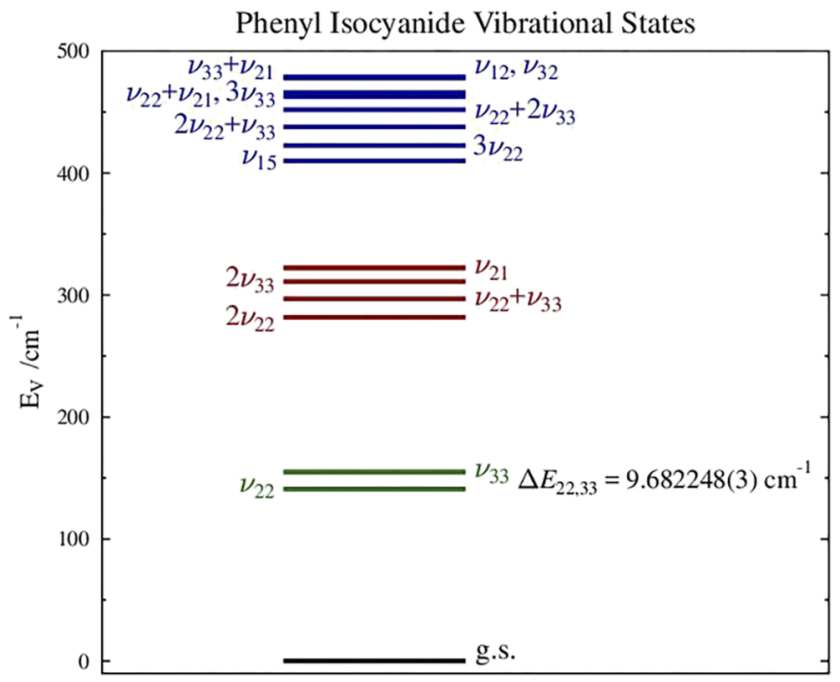

FIG. 4. The vibrational energy levels of phenyl isocyanide below $500 \mathrm{~cm}^{-1}$, drawn from fundamental frequency values of the B3LYP/6-311+G(2d,p) calculation. The $v_{33}-v_{22}$ energy difference results from the perturbation analysis of the present work. 
$\left(J^{\prime \prime}+1=60\right)$ and turnaround (after $\left.J^{\prime \prime}+1=56\right)$ occur at higher frequency than shown in the figure. At high frequencies, $v_{33}$ behaves similarly to $v_{22}$, beginning to progress away from the bandhead toward lower frequency, proceeding through two turnarounds, and eventually progressing to lower frequency. As the values of $C_{v}$ for both of these vibrational states are about $2 \mathrm{MHz}$ greater than those of $C_{0}$, their bands are spaced only slightly more distantly than those of the ground state, with those of $v_{22}$ growing more distant from the ground state than those of $v_{33}$ with increasing $J$. Because the effective inertial defects of the fundamentals, $v_{22}$ and $v_{33}\left[\Delta_{i}=-0.223933(25)\right.$ and $0.378085(15)$, respectively], are substantially larger in magnitude than that of ground vibrational state $\left[\Delta_{i}=0.073921(28)\right]$, the transitions of the vibrational states spread out much more dramatically than those of the ground state.
Our initial approach to the assignment of $v_{22}$ and $v_{33}$ of phenyl isocyanide was the same as the one we employed to assign the analogous dyad of benzonitrile. Namely, we used as initial values the experimentally determined ground-state distortion constants. The initial values of the rotational constants were estimated by applying the B3LYP-predicted vibration-rotation interaction constants $(\alpha$, values) to the experimentally determined ground-state rotational constants. We found, however, that it was remarkably more challenging to obtain an initial fit of the low $K_{a}$ series as a result of the broad spacing of proximal $K_{a}$ series of phenyl isocyanide. Singlestate models were only able to predict the $K_{a}=0,1$ series, so a coupled-state model was immediately implemented. The initial value of the energy separation of the coupled states ( $v_{22}$ and $\left.v_{33}\right), \Delta E$, was taken from the B3LYP anharmonic frequency calculation. As in the case of benzonitrile, phenyl isocyanide is of $C_{2 v}$ symmetry; $v_{22}$

TABLE II. Experimentally determined parameters for $v_{22}$ and $v_{33}$ excited vibrational states of phenyl isocyanide $\left(\mathrm{C}_{6} \mathrm{H}_{5} \mathrm{NC}\right)$ compared to those for the ground state of phenyl isocyanide and the analogous excited states for benzonitrile $\left(\mathrm{C}_{6} \mathrm{H}_{5} \mathrm{CN}\right)$.

\begin{tabular}{|c|c|c|c|c|c|}
\hline & $\begin{array}{l}\text { Phenyl isocyanide } \\
\text { ground state }\end{array}$ & $\begin{array}{l}\text { Phenyl isocyanide } \\
v_{22}\left(B_{1}, 141 \mathrm{~cm}^{-1}\right)\end{array}$ & $\begin{array}{l}\text { Phenyl isocyanide } \\
v_{33}\left(B_{2}, 155 \mathrm{~cm}^{-1}\right)\end{array}$ & $\begin{array}{c}\text { Benzonitrile } \\
v_{22}\left(B_{1}, 141 \mathrm{~cm}^{-1}\right)\end{array}$ & $\begin{array}{c}\text { Benzonitrile } \\
v_{33}\left(B_{2}, 163 \mathrm{~cm}^{-1}\right)\end{array}$ \\
\hline$A_{v}(\mathrm{MHz})$ & $5659.3374(13)$ & $5656.8549(13)$ & [5 659.337375299$]$ & $5654.5(20)$ & $5654.8(20)$ \\
\hline$B_{v}(\mathrm{MHz})$ & $1639.771667(59)$ & $1641.718557(35)$ & $1643.302423(35)$ & $1548.621160(52)$ & $1549.725942(47)$ \\
\hline$C_{v}(\mathrm{MHz})$ & $1271.154548(49)$ & $1273.153282(41)$ & $1272.300426(43)$ & $1216.238170(44)$ & $1215.222889(52)$ \\
\hline$\Delta_{J}(\mathrm{kHz})$ & $0.0494489(40)$ & $0.0502869(36)$ & $0.0507384(40)$ & $0.0459950(27)$ & $0.0461715(27)$ \\
\hline$\Delta_{J K}(\mathrm{kHz})$ & $0.912873(36)$ & $0.91785(29)$ & $0.86729(29)$ & $0.94708(40)$ & $0.90714(40)$ \\
\hline$\Delta_{K}(\mathrm{kHz})$ & $0.2605(17)$ & {$[0.2605]^{a}$} & {$[0.2605]^{\mathrm{a}}$} & {$[0.2423]^{a}$} & {$[0.2423]^{a}$} \\
\hline$\delta_{J}(\mathrm{kHz})$ & $0.0124650(22)$ & $0.01254604(62)$ & $0.01295394(58)$ & $0.01108369(59)$ & $0.01136762(49)$ \\
\hline$\delta_{K}(\mathrm{kHz})$ & $0.60134(13)$ & $0.59457(11)$ & $0.59838(13)$ & $0.607315(93)$ & $0.612108(91)$ \\
\hline$\Phi_{J}(\mathrm{~Hz})$ & $0.00000262(17)$ & $0.00000282(12)$ & $0.00000280(14)$ & $0.000001166(86)$ & $0.000000675(89)$ \\
\hline$\Phi_{J K}(\mathrm{~Hz})$ & $0.0018134(85)$ & $0.0017819(89)$ & $0.0017150(89)$ & $0.0013917(22)$ & $0.0016545(22)$ \\
\hline$\Phi_{K J}(\mathrm{~Hz})$ & $-0.009494(31)$ & $-0.00983(17)$ & $-0.00820(17)$ & $-0.00619(12)$ & $-0.00907(12)$ \\
\hline$\phi_{J K}(\mathrm{~Hz})$ & $0.0008555(58)$ & $0.0007784(51)$ & $0.0007617(58)$ & $0.0007493(41)$ & $0.0006727(42)$ \\
\hline$\Delta E(\mathrm{MHz})$ & & \multicolumn{2}{|c|}{$290266.50(10)^{b}$} & \multicolumn{2}{|c|}{$572848.96(20)^{b}$} \\
\hline$\Delta E\left(\mathrm{~cm}^{-1}\right)$ & & \multicolumn{2}{|c|}{$9.682248(3)$} & \multicolumn{2}{|c|}{$19.108185(7)$} \\
\hline$G_{a}(\mathrm{MHz})$ & & \multicolumn{2}{|c|}{$9718.593(13)$} & \multicolumn{2}{|c|}{$9532.0(62)$} \\
\hline$G_{a}^{J}(\mathrm{MHz})$ & & \multicolumn{2}{|c|}{$-0.0058363(44)$} & \multicolumn{2}{|c|}{$-0.004588(27)$} \\
\hline$G_{a}{ }^{K}(\mathrm{MHz})$ & & \multicolumn{2}{|c|}{$-0.0021119(97)$} & \\
\hline$G_{a}^{J J}(\mathrm{~Hz})$ & & \multicolumn{2}{|c|}{$0.00858(12)$} & & \\
\hline$G_{a}^{J K}(\mathrm{~Hz})$ & & \multicolumn{2}{|c|}{$-0.0760(27)$} & & \\
\hline$F_{b c}(\mathrm{MHz})$ & & \multicolumn{2}{|c|}{$-0.480985(59)$} & \multicolumn{2}{|c|}{$-0.411(39)$} \\
\hline$F_{b c}{ }^{K}(\mathrm{kHz})$ & & \multicolumn{2}{|c|}{$-0.01520(20)$} & \multicolumn{2}{|c|}{$-0.00981(44)$} \\
\hline$\Delta_{i}\left(\mathrm{u} \AA^{2}\right)$ & $0.073921(28)$ & $-0.223933(25)$ & $0.378085(15)$ & $-0.191(32)$ & $0.393(32)$ \\
\hline$N^{\mathrm{c}}$ & 4723 & 3727 & 3627 & 3001 & 2933 \\
\hline$\sigma(\mathrm{MHz})^{\mathrm{d}}$ & 0.034 & 0.040 & 0.040 & 0.034 & 0.037 \\
\hline
\end{tabular}

${ }^{\mathrm{a}} \Delta_{K}$, along with all sextic and octic centrifugal distortion constants not listed explicitly, were fixed at the ground state values of the corresponding molecule from Table I.

${ }^{\mathrm{b}} \Delta E, G_{a}, G_{a}^{J}, G_{a}^{K}, G_{a}^{J}, G_{a}^{J K}, F_{b c}$, and $F_{b c}^{K}$ parameters are for coupling between the $v_{22}$ and $v_{33}$ vibrational states of the corresponding molecule.

${ }^{\mathrm{c}}$ Number of fitted transition frequencies.

${ }^{\mathrm{d}}$ Deviations for the two vibrational state subsets: the overall standard deviation of the coupled fit for phenyl isocyanide to 7354 lines is $0.040 \mathrm{MHz}$ and for benzonitrile to 5934 lines is $0.036 \mathrm{MHz}$. 
and $v_{33}$ belong to $B_{1}$ and $B_{2}$ irreducible representations, respectively. This situation precludes Fermi coupling but allows Coriolis coupling along the inertial axis whose angular momentum component transforms like the cross product of the irreducible representations of the vibrational states: $B_{1} \otimes B_{2}=A_{2}$. The $A_{2}$ irreducible representation corresponds to the $P_{a}$ angular momentum operator so that the appropriate lead spectroscopic constant to represent the Coriolis coupling is $G_{a}$. This term was estimated using the Coriolis coupling constant, $\zeta_{22,33}^{a}$, predicted by the B3LYP calculation. This constant and the spectroscopic constant are directly related by the following equation:

$$
G_{a}=\frac{\omega_{22}+\omega_{33}}{\sqrt{\omega_{22} \times \omega_{33}}} \zeta_{22,33}^{a} A,
$$

where $\omega_{22}$ and $\omega_{33}$ are the harmonic vibrational frequencies for the normal modes and the ground state rotational constant, $A_{0}$, is used for $A$. $^{56}$

Although assignments were made more challenging by the highly spread transitions of the vibrational states, the series were eventually identified and assigned based on the observation of nearcontinuous series in Loomis-Wood plots generated by the AABS software. ${ }^{43}$ These $K_{a}$ series were missing several transitions in the Loomis-Wood plot such that the "gap" moved to higher values of $J^{\prime \prime}$ in subsequent series, a tell-tale pattern of yet-to-be addressed resonances, similar to those observed previously in benzonitrile. After the initial identification of several $K_{a}$ series, the data set was built up by including lines with increasing values of $K_{a}$ in the region below $230 \mathrm{GHz}$, as for the benzonitrile dyad. Initially, only a single rotational constant, $C_{v}$, and the energy separation of the two vibrational states, $\Delta E$, were allowed to vary; additional constants were allowed to be fit with increasing data. The constants were monitored and only allowed to be fit if they remained close to (of the same sign and order of magnitude and not clearly diverging from) the corresponding ground-state value. In the end, the coupling terms $G_{a}, G_{a}^{J}$, $G_{a}^{K}, G_{a}^{J}, G_{a}^{I K}, F_{b c}$, and $F_{b c}^{K}$ (in a previously described expansion of the coupling Hamiltonian), ${ }^{57}$ along with $\Delta E$, were necessary to fit the measured transitions, including resonances and nominal interstate transitions (see below), to within experimental accuracy. The spectroscopic constants determined through this coupled-state analysis are provided in Table II. Data distribution plots for $v_{22}$ and $v_{33}$ are provided in Fig. 5 and demonstrate that lines with high error are scattered throughout the data set, quite like the appearance of the ground vibrational state data distribution plot, suggesting that a fair amount of error in the least-squares fit is due to underlying features. As with the ground vibrational state, there appear to be more high-error measurements along an upward right diagonal. Despite efforts to include additional distortion or coupling terms in the least-squares fit, however, we were unable to eliminate this pattern. The similarity with the ground vibrational state, however, suggests that the same issue may be responsible for the observed pattern in both ground and excited vibrational states.

The $v_{22}$ and $v_{33}$ vibrational states are reported here for the first time; each state contains over 3500 independent measurements. The data sets consist entirely of ${ }^{a} \mathrm{R}_{0,1}$ transitions with $J^{\prime \prime}$ ranging from 42 to 144 and $K_{a}$ ranging from 0 to 48 . While lines with higher values of $K_{a}$ appeared to be visible, the intensities become low and transitions frequently overlap with others, introducing additional unnecessary error into the fit. Thus, these higher $K_{a}$ series were excluded from the data set for least-squares fitting.
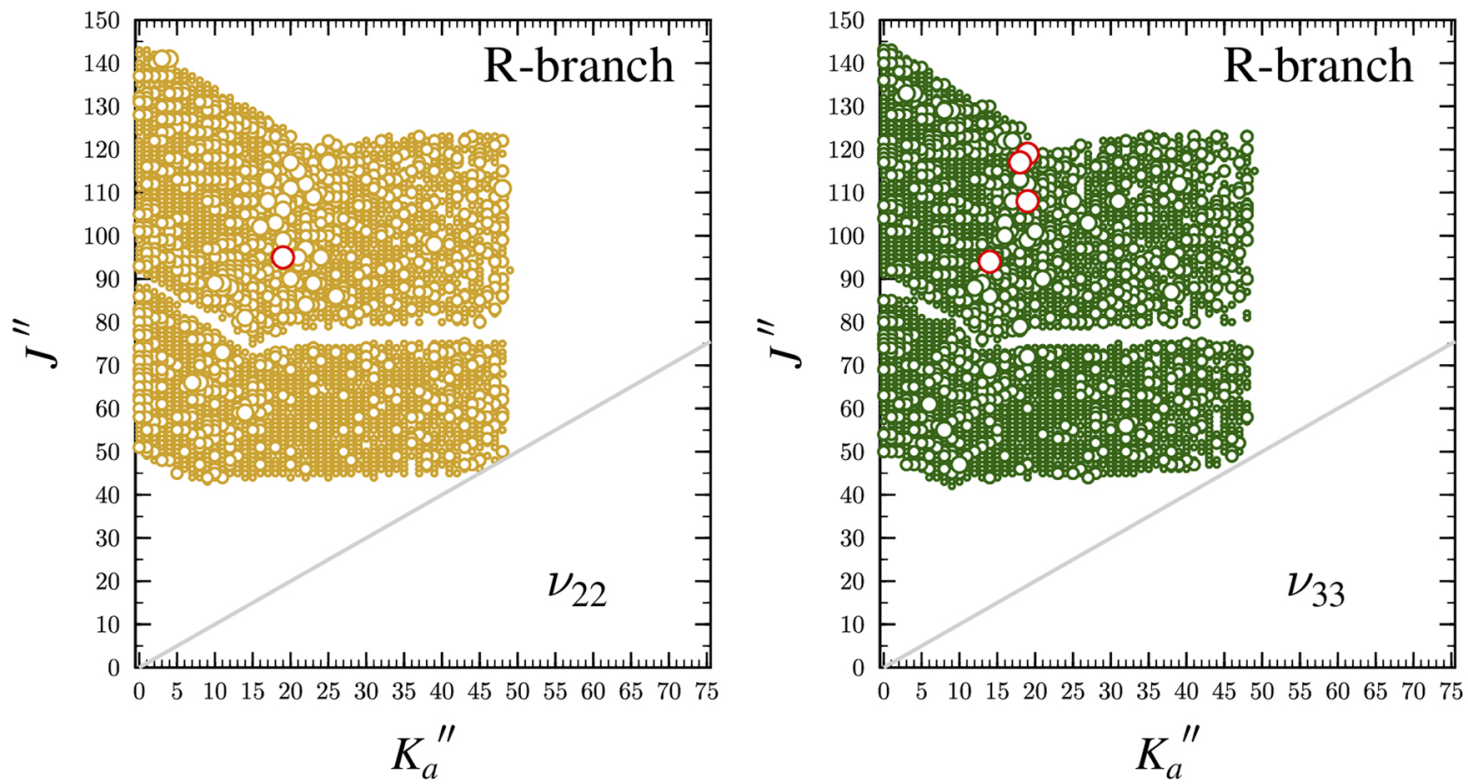

FIG. 5. Data distribution plots of values of $\left|\left(f_{\text {obs. }}-f_{\text {calc. }}\right) / \delta f\right|$ for the coupled fit of measured transitions in the two lowest-energy excited vibrational states in phenyl isocyanide. Transitions with values of $\left|\left(f_{\text {obs. }}-f_{\text {calc. }}\right) / \delta f\right|>3$ are plotted in red. The similarity between the data distribution plots for ground and excited vibrational states suggests that any remaining issues apply to both the single-state and two-state least-squares fits. 
In the final two-state least-squares fit of the dyad, we were unable to determine $A_{v}$ for $v_{33}$. All of the fitted rotational constants are within $0.3 \%$ of the corresponding ground state constant. As in the case of benzonitrile, we were unable to determine $\Delta_{K}$ for either vibrational state due to the availability of only ${ }^{a} \mathrm{R}_{0,1}$ transitions. The other quartic distortion constants, however, are well determined and within $5 \%$ of those of the ground state, indicating that they are likely free of perturbation due to untreated coupling. The three sextic distortion constants that have been determined are within $11 \%$ of those of the ground state.

The inability to determine $A_{v}$ for $v_{33}$ is somewhat perplexing and disappointing, as we were able to determine both values of $A_{v}$ for the corresponding benzonitrile dyad. The values of $A_{22}$ are approximately $2.4 \mathrm{MHz}$ apart, slightly less than the difference in the $A_{0}$ values. The differences between the isomers' rotational constants for $v_{22}$ and $v_{33}$ are otherwise nearly the same as the differences between the ground states. This indicates that the isomers have very similar $a_{\mathrm{i}}$ values. The distortion constants that have been determined are also similar between phenyl isocyanide and benzonitrile. Of the quartic centrifugal distortion constants that were determined, the purely $J$ dependent terms are most dissimilar ( $12 \%$ difference) and values of the purely $K$-dependent term $\left(\delta_{K}\right)$ are most similar, in accordance with the pattern observed for the ground vibrational states of the isomers. The pattern of similarity between the sextic constants, however, is not quite as consistent between the vibrational states, possibly indicating an unresolved issue in the two-state fit of phenyl isocyanide.

The experimental energy separation determined between $v_{22}$ and $v_{33}\left[\Delta E_{22,33}=9.682248(3) \mathrm{cm}^{-1}\right]$ is somewhat smaller than the energy difference predicted using either the B3LYP-predicted harmonic vibrational frequencies $\left(\Delta E_{22,33}=\sim 13.2 \mathrm{~cm}^{-1}\right)$ or the B3LYP-predicted fundamental vibrational frequencies $\left(\Delta E_{22,33}\right.$ $\left.=\sim 14.1 \mathrm{~cm}^{-1}\right)$. The spectroscopically determined Coriolis coupling coefficient $\left(\zeta_{22,33}^{a}=0.858(9)\right)$, however, is very close (within $0.3 \%$ ) to the corresponding value predicted by the vibrational frequency calculation at the B3LYP/6-311+G(2d,p) level of theory $\left(\left|\zeta_{22,33}^{a}\right|=0.8556\right)$.

\section{Interpretation and analysis of the resonances}

Once transitions furthest removed from the resonances had been assigned and fit, it became possible to assign lines closer and closer to the major resonances. By iteratively adding lines closer to the center of the resonance, refitting, and re-predicting, we were able to assign numerous resonances for this dyad. The resonances predominantly follow a $\Delta K_{a}=2$ and $4\left(\Delta K_{c}=3\right)$ selection rule, as in benzonitrile. The resonance plots of a pair of corresponding resonances with $\Delta K_{a}=2$ are provided in Fig. 6, showing the mirror image pattern that confirms their assignment. The magnitude of the resonances (the displacement of the furthest point on the resonance from its predicted frequency in the absence of local perturbation) can be gauged using the $y$-axis of the resonance plots $\left[\left(v-v_{0}\right) /\right.$ $\left.\left(J^{\prime \prime}+1\right)\right]$. The largest resonances between $v_{22}$ and $v_{33}$ of phenyl isocyanide are notably larger than those of benzonitrile. A set of $\Delta K_{a}=2$ resonances for each molecule is provided in Fig. 7. The axes are set to the same scale so that it is easy to compare the magnitude of the resonances. The resonances provided for benzonitrile in this figure are two of the largest in magnitude that we observed in the

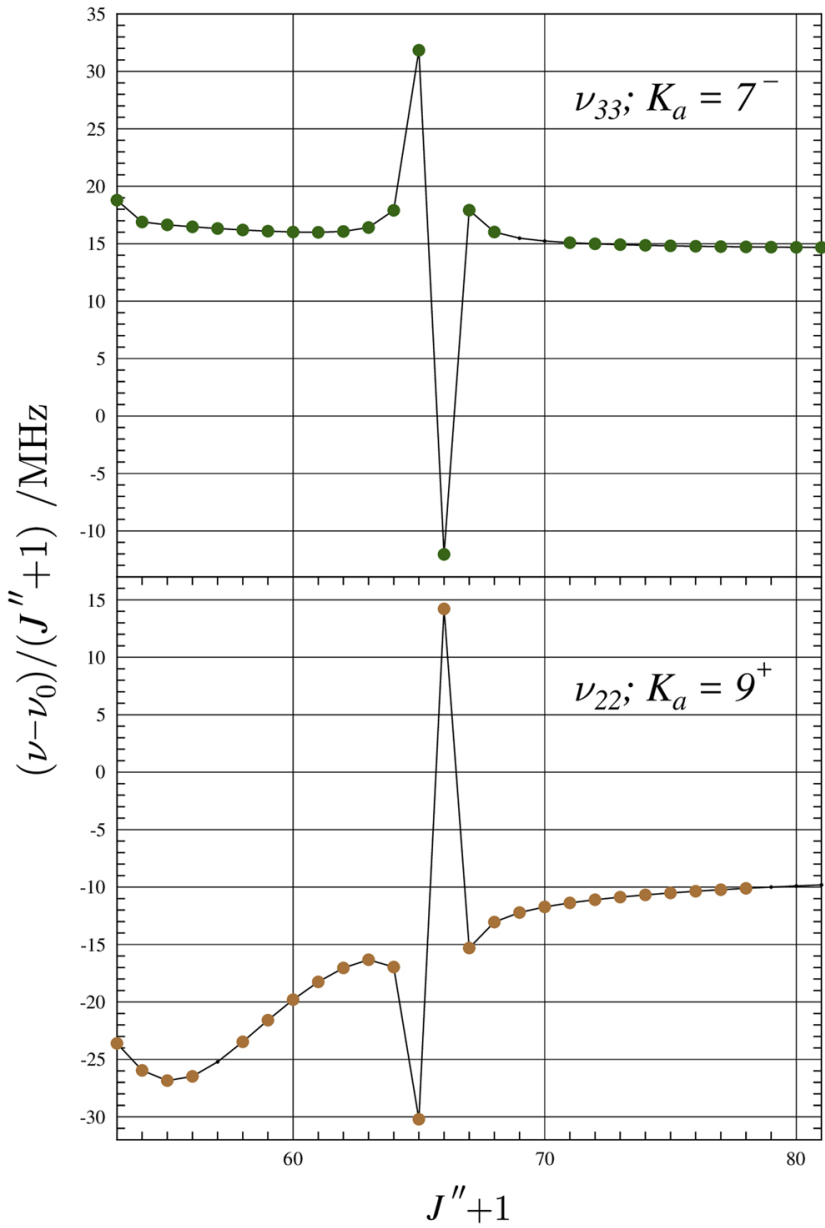

FIG. 6. Resonance plots for phenyl isocyanide showing the $K_{a}=9^{+}$series for $v_{22}$ and $K_{a}=7^{-}$series for $v_{33}$, an example of resonances conforming to the $\Delta K_{a}=2$ selection rule. The plotted values are frequency differences between excited state transitions and their ground state counterparts, scaled by $\left(J^{\prime \prime}+1\right)$ in order to make the plots more horizontal. Measured transitions are represented by circles: $v_{22}$ (copper) and $v_{33}$ (green). There are no measured transitions with $\left|\left(f_{\text {obs. }}-f_{\text {calc. }}\right) / \delta f\right|>3$. Predictions from the final coupled fit are represented by a solid, black line. The two resonances are mirror images of one another, confirming the $K_{a}$ assignment of these resonance partners.

103-360 GHz spectral region, while those displayed for phenyl isocyanide are of medium magnitude relative to its largest resonances. It is evident from the $y$-axis in Fig. 7 that the most displaced point of the phenyl isocyanide resonance $\left(\right.$ at $J^{\prime \prime}+1=62$ ) appears approximately 19 units from its expected position absent local perturbation, which translates to $\sim 1200 \mathrm{MHz}\left(v-v_{0}\right)$. The largest resonances of phenyl isocyanide appear up to $\sim 9400 \mathrm{MHz}$ $\left(v-v_{0}\right)$ away from where they would be expected based on the frequencies of other transitions in the corresponding $K_{a}$ series. By comparison, the largest resonances we observed for benzonitrile between the analogous dyad of states were displaced from the expected frequency by less than $600 \mathrm{MHz}\left(v-v_{0}\right)$. This trend of larger resonances in the dyad of phenyl isocyanide is 

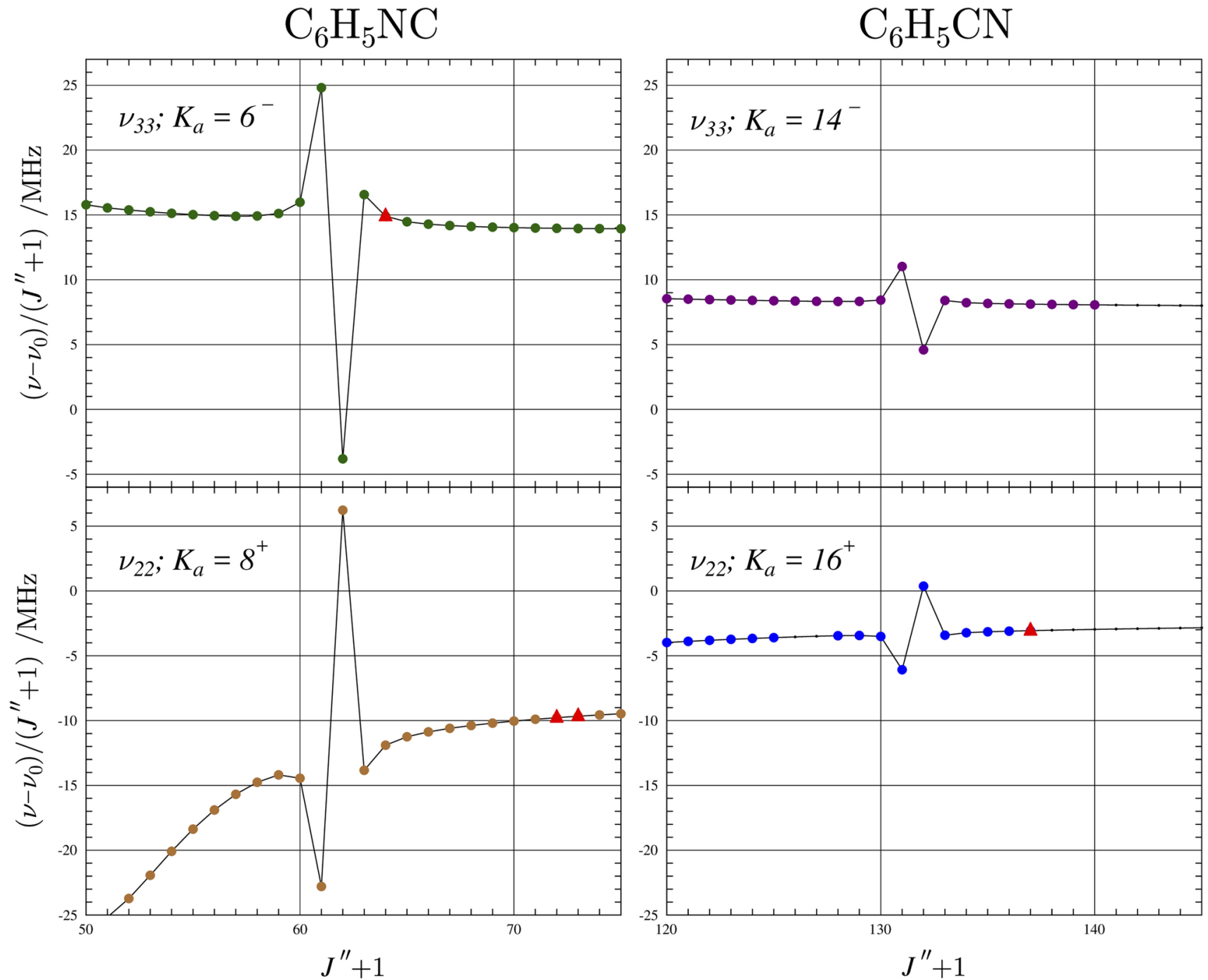

FIG. 7. Resonance plots showing $K_{a}=8^{+}$for $v_{22}$ and $K_{a}=6^{-}$for $v_{33}$ of phenyl isocyanide (left) and $K_{a}=16^{+}$for $v_{22}$ and $K_{a}=14^{-}$for $v_{33}$ of benzonitrile (right). The plotted values are frequency differences between excited state transitions and their ground state counterparts, scaled by $\left(\mathrm{J}^{\prime \prime}+1\right)$ in order to make the plots more horizontal. Measured transitions are represented by circles: $v_{22}$ of phenyl isocyanide (copper), $v_{33}$ of phenyl isocyanide (green), $v_{22}$ of benzonitrile (blue), and $v_{33}$ of benzonitrile (purple). Measured transitions with $\left|\left(f_{\text {obs. }}-f_{\text {calc. }}\right) / \delta f\right|>3$ are marked with red triangles. Predictions from the final coupled fit are represented by a solid, black line. The $x$ - and $y$-axes are set to the same scale for each of the resonance plots.

illustrated globally in Fig. 8, which shows a set of superimposed resonance plots for $K_{a}{ }^{+}$series (even values of $K_{a}$ between 2 and 30 are included for clarity); Fig. 8(a) is phenyl isocyanide and Fig. 8 (b) is the corresponding set of resonance plots for benzonitrile. Whereas the reference state in Figs. 6 and 7 is the ground vibrational state, the reference state in Fig. 8 is a deperturbed prediction using the final coupled-state fit rotational and distortion constants, but with the perturbation parameters set to zero. These resonance plots thus show purely the effect of perturbation and not any effects due to differences between rotational and distortion constants between the vibrational and ground states. These resonance plots do look highly similar to those using the ground state as a reference, validating such ground state-referenced plots. The resonance plots in Fig. 8 show two obvious features: the resonances already discussed and the broader undulation due to global perturbation. It can be seen that there is a regular progression of the resonance position and amplitude as $K_{a}$ increases. Furthermore, it is evident that the resonance amplitudes for phenyl isocyanide are approximately 15 times larger than those in benzonitrile for matching values of $K_{a}$. Initially, this may seem surprising. Although the energy separation between vibrational states is smaller in the dyad of phenyl isocyanide than in benzonitrile, the energy separation 


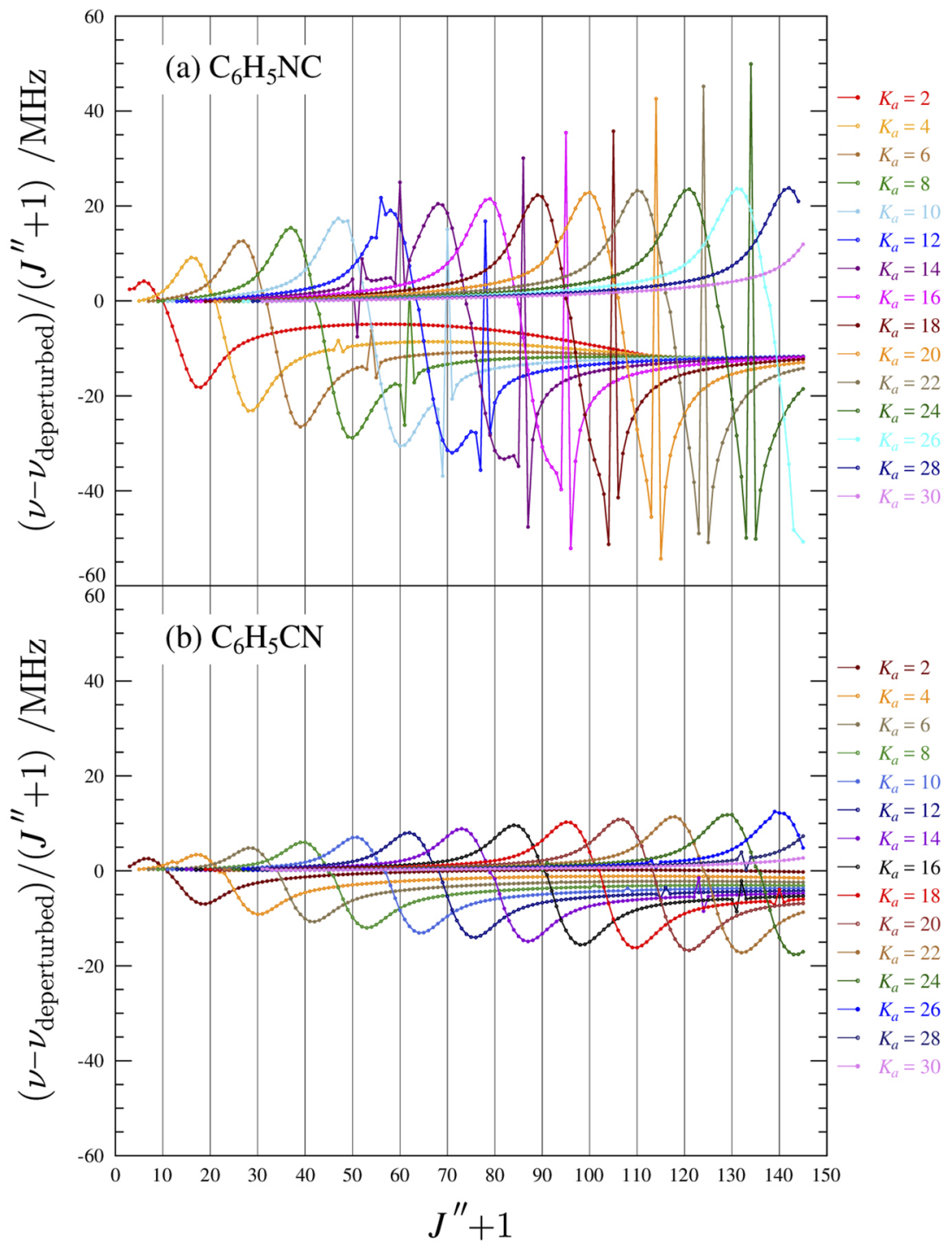

FIG. 8. Superimposed resonance plots of $v_{22}$ for $K_{a}{ }^{+}$series with even values of $K_{a}$ between 2 and 30 for phenyl isocyanide (a) and benzonitrile (b). Measured transitions are omitted for clarity, but they are indistinguishable from the plotted values on this scale. The plotted values are frequency differences between excited state transitions and their deperturbed counterparts, scaled by $\left(J^{\prime \prime}+1\right)$, as for Figs. 6 and 7. The $x$ - and $y$-axes are set to the same scale for each of the resonance plots.

between actual interacting rovibrational states is scattered in a not easily predictable pattern but with similar magnitudes. Figure 9(a) shows the $K_{a}=14^{+}$and $14^{-}$energy level perturbations for $v_{22}$ and $v_{33}$ of phenyl isocyanide. Figure 9 (b) shows the four corresponding resonance plots, which are essentially each a finite difference first derivative curve of the energy curves in Fig. 9(a). The resonance plots in Fig. 9(b) are like those in Fig. 8 but are not scaled by $\mathrm{J}^{\prime \prime}+1$. In these plots, the mirroring of global perturbation $\left(\Delta K_{a}=0\right)$ is apparent, while the resonances are offset due to their differing selection rules. Three distinct regions are evident in these plots. The first is a low $J$ region where the molecule behaves as a prolate top and the perturbation in Fig. 9(a) is fairly constant, approximately equal to the simple two-state model result shown in the following equation:

$$
\sqrt{\left(\frac{\Delta E_{22,33}}{2}\right)^{2}+G_{a}^{2} K_{a}^{2}}-\frac{\Delta E_{22,33}}{2}
$$

The second region, where the molecule behaves asymmetrically, appears as an undulation at medium values of $J$. Since the undulations in Fig. 9(b) are the derivatives relative to the asymmetry curve, their position depends on the asymmetry parameter $\left(\kappa=\frac{2 B-A-C}{A-C}\right)$ and not on $\Delta E_{22,33}$. The values of $\kappa$ are very similar between the two isomeric molecules. It is worth noting that the strongest resonances in Fig. 8 appear to track at least approximately with the undulation, indicating that the level of asymmetry also largely influences their positions. Finally, there is a high $J$ region where the molecule behaves as an oblate top, where $K_{c}$ becomes nearly a good quantum number and the perturbation magnitude increases approximately quadratically with $J$. The very strong scaling of the resonances with $\Delta \mathrm{E}$ is evidently related to an interaction of the very large global $\left(\Delta K_{a}\right.$ $=0)$ perturbation with smaller, local perturbations $\left(\Delta K_{a}=2\right.$ or 4$)$ in the matrix diagonalization. Contour plots of phenyl isocyanide and benzonitrile are depicted in Fig. 10. It is evident from these plots that 


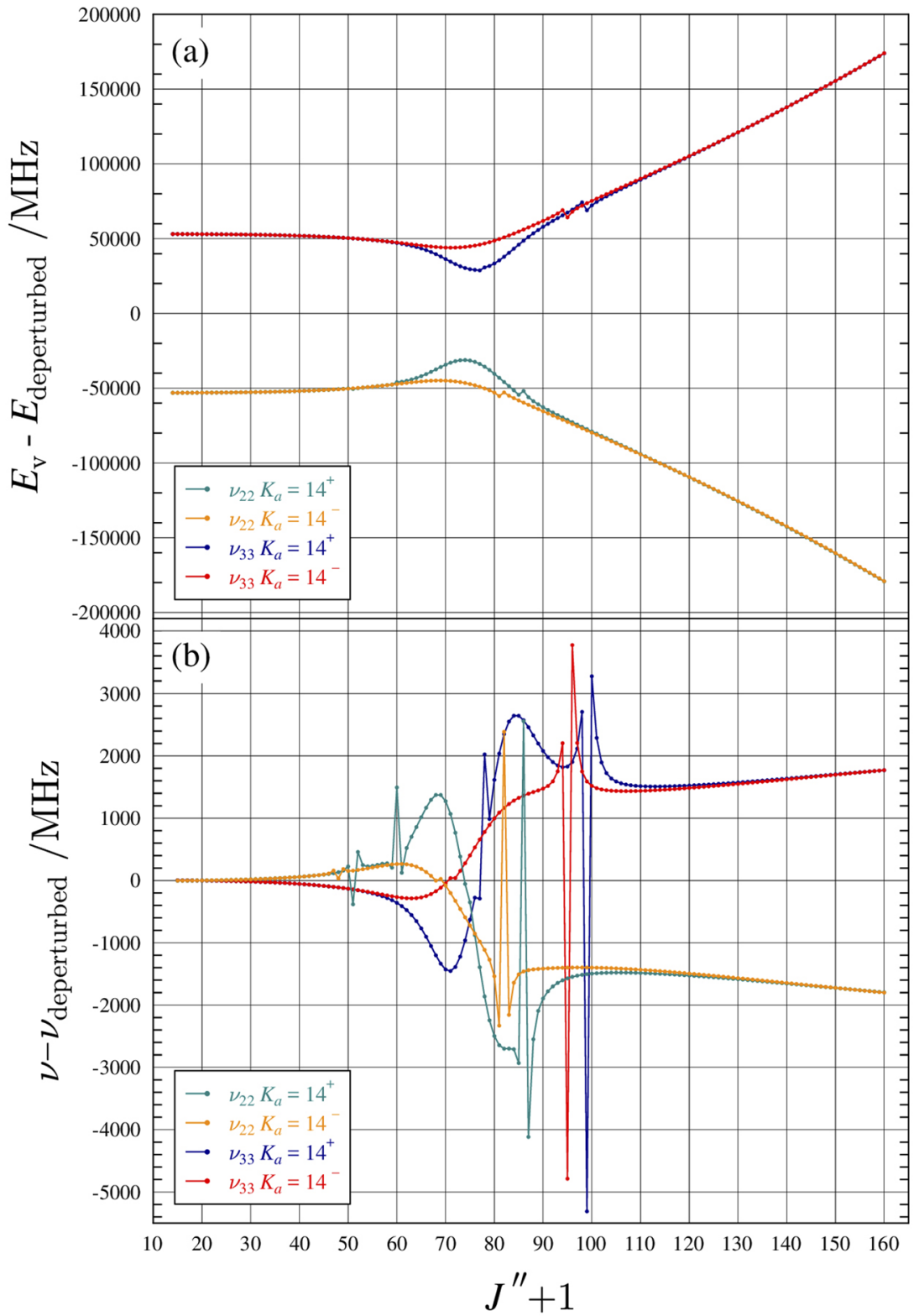

FIG. 9. Energy difference plots for $K_{a}=14$ of phenyl isocyanide obtained by subtracting the deperturbed energies from the final coupled-fit energies (a) and corresponding frequency difference plots (b). Measured transitions are omitted for clarity in (b), but they are indistinguishable from the plotted values on this scale. The $x$-axis is set to the same scale for each of the plots and the same color scheme is used in (a) and (b).

resonances in phenyl isocyanide appear at lower values of $J^{\prime \prime}$ and $K_{a}{ }^{\prime \prime}$ than in benzonitrile. Again, this behavior is reasonable based upon the smaller energy separation between $v_{22}$ and $v_{33}$ in phenyl isocyanide, allowing the energy levels of the lower state to intercept those of the upper state at lower $K_{a}$.

As in benzonitrile, the final least-squares fit allowed the prediction and measurement of several nominal interstate transitions. Such formally forbidden transitions are enabled by strong mixing between rotational levels of different vibrational states. All nominal interstate transitions included in the least-squares fit are provided in the supplementary material, and a representative "matched pair" of nominal interstate transitions is illustrated in Fig. 11.
A "matched pair" consists of nominal interstate transitions whose lower and upper rotational levels correspond to the lower and upper rotational levels of two standard, within-state ${ }^{a} \mathrm{R}_{0,1}$ transitions. Because phenyl isocyanide exhibits stronger resonances than does benzonitrile, there was a larger number of strong nominal interstate transitions predicted and measured for phenyl isocyanide. Initially, 42 matched pairs were identified as candidates for inclusion in the least-squares fit for phenyl isocyanide. To be included in the final least-squares fit, however, the measured nominal interstate transitions had to meet several criteria: (1) the transitions had to have similar intensities in the rotational spectrum, (2) all four transitions - the pair of nominal interstate transitions and the two 

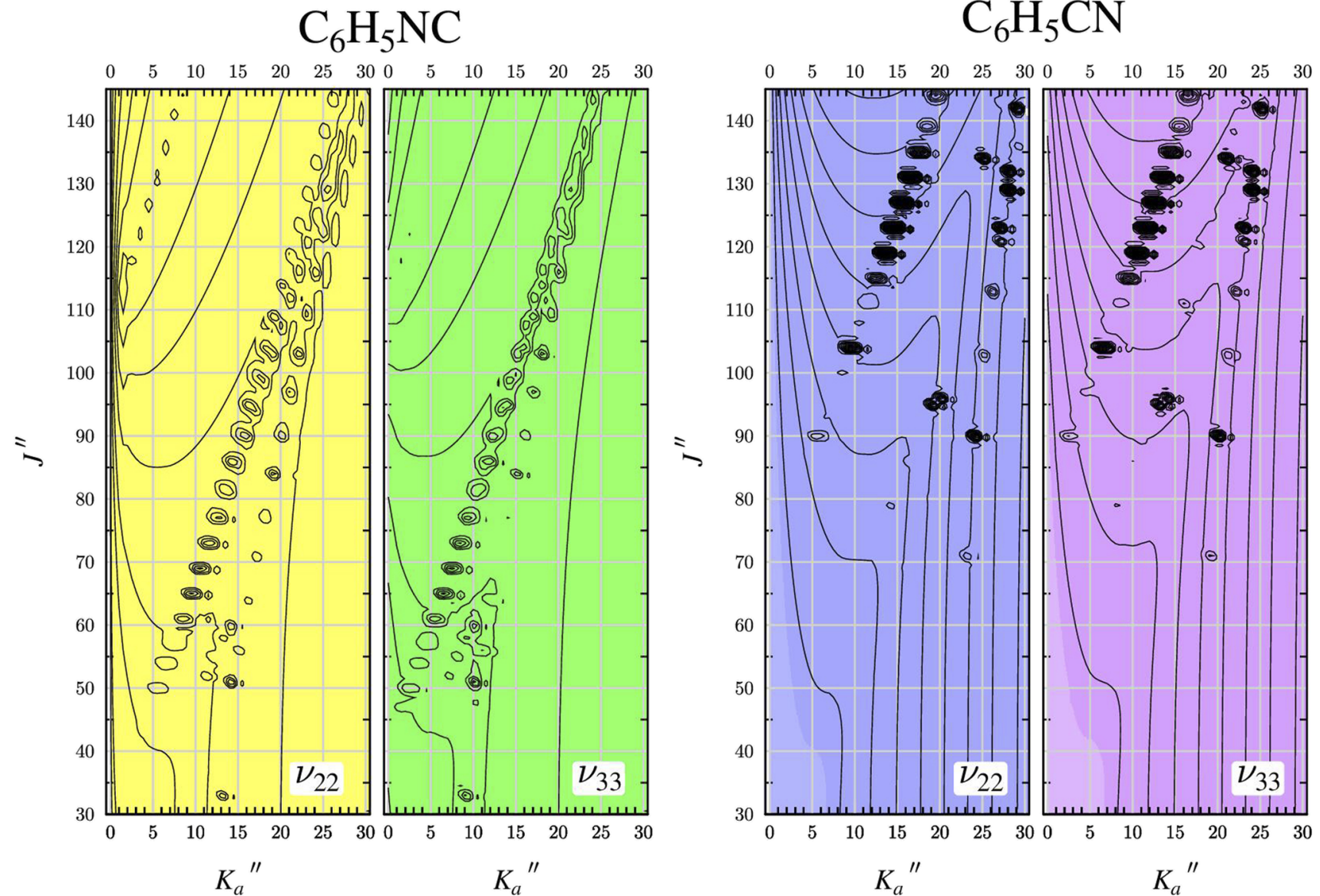

FIG. 10. Contour plots depicting the coupling landscape between rotational levels in $v_{22}$ and $v_{33}$ vibrational states of phenyl isocyanide (left: yellow and green, respectively) and benzonitrile (right: blue and purple, respectively). The mapped values are $\left(1-P_{\text {mix }}\right)$, where $P_{\text {mix }}$ is the mixing coefficient of a given vibration-rotation energy level. Resonances between levels in the two vibrational states are apparent as matching, similarly shaped islands along the horizontal direction (same $J$ ) but differing in the values of $K_{a}$ (see text). The horizontally elongated resonance islands appear to be resonances for $\Delta K_{a}=3$ but are in fact sums of $\Delta K_{a}=2$ and $\Delta K_{a}=4$ resonances.

corresponding within-state transitions-had to be incorporated into the overall data set, and (3) the average of the frequencies of the nominal interstate matched pair had to be equal within their combined measurement errors $(\sim 0.07 \mathrm{MHz})$ to the average of the corresponding within-state frequencies. As a result, we included only 14 of the 42 matched pair candidates in the final least-squares fit. Using the same selection criteria, a total of 11 matched pairs were included in the final least-squares fit for benzonitrile. It is worth noting that nearly all of the nominal interstate transitions measured for benzonitrile are degenerate pairs of transitions, resulting in a roughly two-fold increase to the intensity of each of these transitions. All of the nominal interstate transitions measured for phenyl isocyanide are nondegenerate transitions. Thus, their intensity does not benefit from the intensity boost, and yet they are still sufficiently intense to be observed, again attesting to the stronger intensity of the individual nominal interstate transitions of the isocyanide isomer.

\section{Comparison with computational estimates}

Table III provides a comparison between experimentally determined values for the least-squares fit for $v_{22}$ and $v_{33}$ dyad with their B3LYP-estimated values. The experimentally determined $b$ - and $c$ axis vibration-rotation interaction constants $\left(B_{0}-B_{v}\right.$ and $\left.C_{0}-C_{v}\right)$ for both vibrational states are in excellent agreement with their B3LYP-predicted values. The errors in the computational predictions are remarkably small, $2 \%$ or less for each of these values. Unfortunately, $A_{0}-A_{33}$ was unable to be determined in this work. The value of $A_{0}-A_{22}$ has the greatest difference with the calculated value of any of the vibration-rotation interaction constants. While $A_{0}-A_{22}$ was too low by about $80 \%$ of its value, the discrepancy of about $2 \mathrm{MHz}$ is still quite small compared to the value of $A_{22}$, 5656.8549 (13) $\mathrm{MHz}$. It is not surprising that the $A$ constant has the largest discrepancy with its calculated value as it is the largest constant and the constant most correlated with the $K$-dependent 


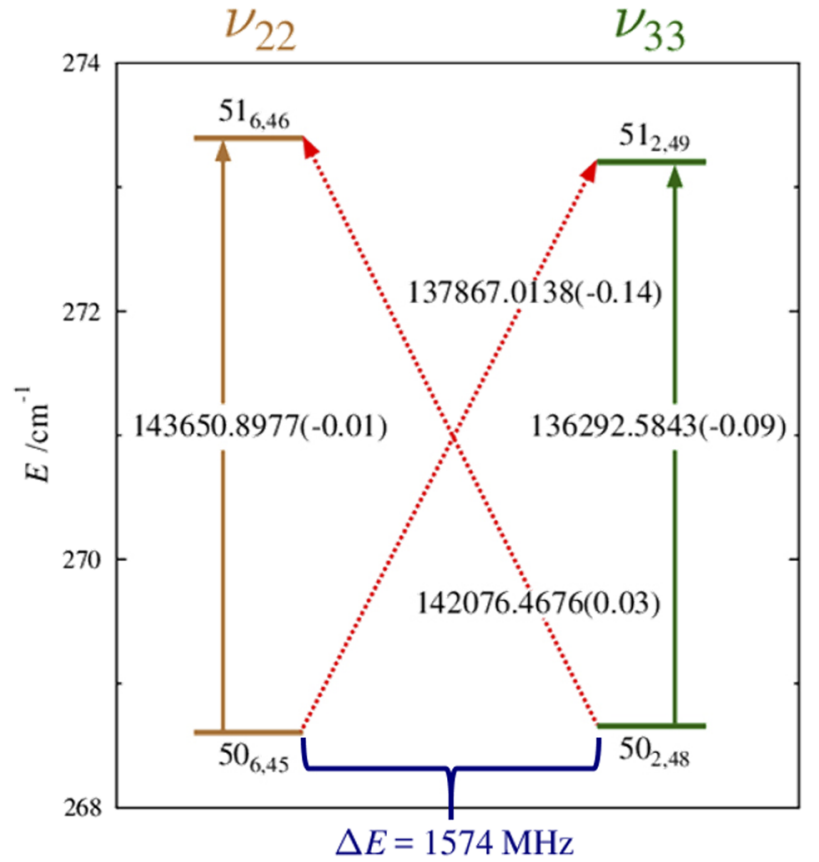

FIG. 11. Energy diagram depicting a representative matched pair of nominal rotation-vibration transitions between $v_{22}$ (copper) and $v_{33}$ (green) vibrational states of phenyl isocyanide. Standard ${ }^{a} \mathrm{R}_{0,1}$ transitions within vibrational states are denoted by vertical arrows. The diagonal, dashed arrows indicate nominal interstate transitions that are formally forbidden but observed as a result of rotational level mixing. The value printed on each arrow is the corresponding transition frequency (in $\mathrm{MHz}$ ) with its observed-calculated value in parentheses. The marked energy separation is the energy separation between the two strongly interacting rotational energy levels.

distortion constants $\left(\Delta_{K}\right.$ and $\left.\Phi_{K}\right)$ and the Coriolis coupling terms. It is also expected to be strongly correlated with the undetermined value of $A_{33}$. As mentioned previously, the quartic and sextic distortion constants and the Coriolis coupling constants were very well predicted by the B3LYP calculation. Collectively, the predicted values from the B3LYP calculation provided excellent support to the experimental work.

TABLE III. Experimental and computed vibration-rotation interaction constants $\left(\alpha_{i}\right)$ for $v_{22}$ and $v_{33}$ excited vibrational states of phenyl isocyanide $\left(\mathrm{C}_{6} \mathrm{H}_{5} \mathrm{NC}\right)$.

\begin{tabular}{lcc}
\hline \hline & Experimental & B3LYP/6-311+(2d,p) \\
\hline & $v_{22}$ & \\
\hline$A_{0}-A_{22}(\mathrm{MHz})$ & $2.4825(18)$ & 4.505 \\
$B_{0}-B_{22}(\mathrm{MHz})$ & $-1.946890(69)$ & -1.986 \\
$C_{0}-C_{22}(\mathrm{MHz})$ & $-1.998734(64)$ & -2.023 \\
\hline & $v_{33}$ & \\
\hline$A_{0}-A_{33}(\mathrm{MHz})$ & & -2.050 \\
$B_{0}-B_{33}(\mathrm{MHz})$ & $-3.530756(69)$ & -3.461 \\
$C_{0}-C_{33}(\mathrm{MHz})$ & $-1.145878(65)$ & -1.134 \\
\hline \hline
\end{tabular}

\section{CONCLUSIONS}

The present extensive data set and analysis of the ground vibrational state and two lowest-energy vibrational states of phenyl isocyanide have enabled a detailed comparison of its spectrum and behavior to those of benzonitrile. In particular, this is the first time a comparison of the Coriolis perturbation parameters for two such closely related isomers has been made. While the overall spectroscopic constants have proven to be very similar, there exist notable differences due to the smaller energy separation between $v_{22}$ and $v_{33}$ for phenyl isocyanide. This value is approximately half that for benzonitrile. As a result, the observed resonances are shifted to lower quantum number values. The magnitudes of the broad undulations are observed to scale roughly as the inverse of the energy separation, but those of the observed resonances vary by a much larger factor. While we were surprisingly unable to determine $A_{33}$, the perturbation analysis for phenyl isocyanide did permit determination of three additional higher-order coupling terms $\left(G_{a}^{K}, G_{a}^{J J}\right.$, and $G_{a}^{K K}$ ) that were not determined for benzonitrile, even though the data sets were comparable in size. The analysis of numerous resonances allowed for a precise determination of the energy difference of $9.682248(3) \mathrm{cm}^{-1}$ between the $v_{22}$ and $v_{33}$ fundamental states of phenyl isocyanide. This work supports the conclusion that B3LYP/6$311+(2 \mathrm{~d}, \mathrm{p})$ anharmonic frequency calculations provide very useful estimates of the equilibrium rotational constants, fourth- and sixthorder centrifugal distortion constants, vibration-rotation interaction constants, and first-order Coriolis coupling constants. The expanded spectroscopic data set and the much more complete analysis of centrifugal distortion now permit prediction of all rotational transitions accessible to radioastronomy to sufficient precision for astronomical detection and identification.

\section{SUPPLEMENTARY MATERIAL}

See supplementary material for least-squares fitting files of phenyl isocyanide, output files from computations, computed vibration-rotation interaction constants, computed vibrational frequencies and infrared intensities, nominal interstate transitions for the $v_{22}$ and $v_{33}$ dyad of phenyl isocyanide with their corresponding within-state transitions, and the NMR and mass spectra of phenyl isocyanide.

\section{ACKNOWLEDGMENTS}

We gratefully acknowledge funding from the National Science Foundation for support of this project (No. NSF-1664912) and for support of shared departmental computing resources (Grant No. NSF-CHE-0840494). We also gratefully acknowledge funding from the National Institutes of Health for support of the shared departmental mass spectrometry instruments (Grant No. NIH 1S10OD020022-1). M.A.Z. thanks Andrew Maza for his assistance and instruction in the phenyl isocyanide synthesis. We thank Michael McCarthy for the loan of an Amplification-Multiplication Chain and Mark Wendt for the loan of an analog signal generator. We thank the Harvey Spangler Award (to B.J.E.) for the funding that supported the purchase of the Virginia Diodes zero-bias detector. Finally, we extend our gratitude to Zbigniew Kisiel for his insightful discussions on the coupled dyad fit of benzonitrile, which greatly facilitated this work on its isomer. 


\section{REFERENCES}

${ }^{1}$ B. A. McGuire, A. M. Burkhardt, S. V. Kalenskii, C. N. Shingledecker, A. J. Remijan, E. Herbst, and M. C. McCarthy, "Detection of the aromatic molecule benzonitrile $\left(c-\mathrm{C}_{6} \mathrm{H}_{5} \mathrm{CN}\right)$ in the interstellar medium," Science 359, 202-205 (2018).

${ }^{2}$ N. Kaifu, M. Ohishi, K. Kawaguchi, S. Saito, S. Yamamoto, T. Miyaji, K. Miyazawa, S.-i. Ishikawa, C. Noumaru, S. Harasawa, M. Okuda, and H. Suzuki, "A 8.8-50 GHz complete spectral line survey toward TMC-1. Part 1. Survey data," Publ. Astron. Soc. Jpn. 56, 69-173 (2004).

${ }^{3}$ B. Bak, B. P. Van Eijck, and C. Kierkegaard, "On the structure of phenylisocyanide," J. Mol. Struct. 18, 429-434 (1973).

${ }^{4}$ W. Kasten, H. Dreizler, and U. Andresen, "Nitrogen quadrupole coupling in the microwave ground state spectra of tertiary-butyl isocyanide and phenyl isocyanide," Z. Naturforsch. A 41, 1302-1306 (1986).

${ }^{5} \mathrm{~B}$. Bak and C. Kierkegaard, "Infrared and Raman spectra of phenylisocyanide $\mathrm{C}_{6} \mathrm{H}_{5} \mathrm{~N} \equiv \mathrm{C}$," Acta Chem. Scand. 27, 2531-2535 (1973).

${ }^{6}$ F. J. Lovas, R. J. McMahon, J.-U. Grabow, M. Schnell, J. Mack, L. T. Scott, and R. L. Kuczkowski, "Interstellar Chemistry: A strategy for detecting polycyclic aromatic hydrocarbons in space," J. Am. Chem. Soc. 127, 4345-4349 (2005).

${ }^{7}$ B. M. Jones, F. Zhang, R. I. Kaiser, A. Jamal, A. M. Mebel, M. A. Cordiner, and S. B. Charnley, "Formation of benzene in the interstellar medium," Proc. Natl. Acad. Sci. U. S. A. 108, 452-457 (2011).

${ }^{8} \mathrm{C}$. Joblin and J. Cernicharo, "Detecting the building blocks of aromatics," Science 359, 156-157 (2018).

${ }^{9} \mathrm{P}$. Thaddeus, J. M. Vrtilek, and C. A. Gottlieb, "Laboratory and astronomical identification of cyclopropenylidene, $\mathrm{C}_{3} \mathrm{H}_{2}$," Astrophys. J. 299, L63-L66 (1985).

${ }^{10} \mathrm{P}$. Cox, R. Guesten, and C. Henkel, "Observations of $\mathrm{C}_{3} \mathrm{H}_{2}$ in the diffuse interstellar medium,” Astron. Astrophys. 206, 108-116 (1988).

${ }^{11}$ J. M. Hollis, A. J. Remijan, P. R. Jewell, and F. J. Lovas, "Cyclopropenone $\left(c-\mathrm{H}_{2} \mathrm{C}_{3} \mathrm{O}\right)$ : A new interstellar ring molecule," Astrophys. J. 642, 933-939 (2006).

${ }^{12}$ J. Cernicharo, A. M. Heras, A. G. G. M. Tielens, J. R. Pardo, F. Herpin, M. Guelin, and L. B. F. M. Waters, "Infrared space observatory's discovery of $\mathrm{C}_{4} \mathrm{H}_{2}, \mathrm{C}_{6} \mathrm{H}_{2}$, and benzene in CRL 618," Astrophys. J. 546, L123-L126 (2001).

${ }^{13}$ J. Cami, J. Bernard-Salas, E. Peeters, and S. E. Malek, "Detection of $\mathrm{C}_{60}$ and $\mathrm{C}_{70}$ in a young planetary nebula," Science 329, 1180-1182 (2010).

${ }^{14} \mathrm{~K}$. Wohlfart, M. Schnell, J.-U. Grabow, and J. Küpper, "Precise dipole moment and quadrupole coupling constants of benzonitrile," J. Mol. Spectrosc. 247, 119121 (2008).

${ }^{15}$ H. S. P. Müller, S. Thorwirth, D. A. Roth, and G. Winnewisser, "The Cologne database for molecular spectroscopy, CDMS," Astron. Astrophys. 370, L49-L52 (2001).

${ }^{16}$ M. Guelin, S. Muller, J. Cernicharo, M. C. McCarthy, and P. Thaddeus, "Detection of the SiNC radical in IRC+10216," Astron. Astrophys. 426, L49-L52 (2004).

${ }^{17}$ M. Guelin, S. Muller, J. Cernicharo, A. J. Apponi, M. C. McCarthy, C. A. Gottlieb, and P. Thaddeus, "Astronomical detection of the free radical SiCN," Astron. Astrophys. 363, L9-L12 (2000).

${ }^{18}$ K. Kawaguchi, E. Kagi, T. Hirano, S. Takano, and S. Saito, "Laboratory spectroscopy of isocyanomagnesium radical: The first radioastronomical identification of Mg-bearing molecule," Astrophys. J. 406, L39-L42 (1993).

${ }^{19} \mathrm{M}$. Guelin, R. Lucas, and J. Cernicharo, "MgNC and the carbon-chain radicals in IRC+10216," Astron. Astrophys. 280, L19-L22 (1993).

${ }^{20}$ L. M. Ziurys, "The chemistry in circumstellar envelopes of evolved stars: Following the origin of the elements to the origin of life," Proc. Natl. Acad. Sci. U. S. A. 103, 12274-12279 (2006).

${ }^{21}$ M. Agundez, J. Cernicharo, P. de Vicente, N. Marcelino, E. Roueff, A. Fuente, M. Gerin, M. Guelin, C. Albo, A. Barcia, L. Barbas, R. Bolano, F. Colomer, M. C. Diez, J. D. Gallego, J. Gomez-Gonzalez, I. Lopez-Fernandez, J. A. LopezFernandez, J. A. Lopez-Perez, I. Malo, J. M. Serna, and F. Tercero, "Probing non-polar interstellar molecules through their protonated form: Detection of protonated cyanogen $\left(\mathrm{NCCNH}^{+}\right)$," Astron. Astrophys. 579, L10 (2015).

${ }^{22} \mathrm{M}$. Agundez, N. Marcelino, and J. Cernicharo, "Discovery of interstellar isocyanogen $(\mathrm{CNCN})$ : Further evidence that dicyanopolyynes are abundant in space," Astrophys. J. 861, L22 (2018).
${ }^{23}$ C. M. Walmsley, G. Winnewisser, and F. Toelle, "Cyanoacetylene and cyanodiacetylene in interstellar clouds," Astron. Astrophys. 81, 245-250 (1980).

${ }^{24}$ H. Suzuki, S. Yamamoto, M. Ohishi, N. Kaifu, S. Ishikawa, Y. Hirahara, and S. Takano, "A survey of $\mathrm{CCS}, \mathrm{HC}_{3} \mathrm{~N}, \mathrm{HC}_{5} \mathrm{~N}$, and $\mathrm{NH}_{3}$ toward dark cloud cores and their production chemistry," Astrophys. J. 392, 551-570 (1992).

${ }^{25} \mathrm{~K}$. Kawaguchi, M. Ohishi, S. Ishikawa, and N. Kaifu, "Detection of isocyanoacetylene HCCNC in TMC-1," Astrophys. J. 386, L51-L53 (1992).

${ }^{26}$ S.-Y. Liu, D. M. Mehringer, and L. E. Snyder, "Observations of formic acid in hot molecular cores," Astrophys. J. 552, 654-663 (2001).

${ }^{27}$ A. Fuente, S. Garcia-Burillo, M. Gerin, D. Teyssier, A. Usero, J. R. Rizzo, and P. de Vicente, "Photon-dominated chemistry in the nucleus of M82: Widespread HOC+ emission in the inner 650 parsec disk," Astrophys. J. 619, L155-L158 (2005).

${ }^{28}$ L. E. Snyder and D. Buhl, "Radio emission from interstellar hydrogen cyanide," Astrophys. J. 163, L47-L52 (1971).

${ }^{29}$ J. H. Bieging, S. Shaked, and P. D. Gensheimer, "Submillimeter- and millimeterwavelength observations of $\mathrm{SiO}$ and $\mathrm{HCN}$ in circumstellar envelopes of AGB stars," Astrophys. J. 543, 897-921 (2000).

${ }^{30}$ P. Schilke, D. J. Benford, T. R. Hunter, D. C. Lis, and T. G. Phillips, "A line survey of Orion-KL from 607 to $725 \mathrm{GHz}$," Astrophys. J., Suppl. Ser. 132, 281-364 (2001).

${ }^{31}$ P. Schilke, C. Comito, and S. Thorwirth, "First detection of vibrationally excited HNC in space,” Astrophys. J. 582, L101-L104 (2003).

${ }^{32}$ B. E. Turner and B. Zuckerman, "Observations of strongly deuterated molecules: Implications for interstellar chemistry," Astrophys. J. 225, L75-L79 (1978).

${ }^{33}$ J. Cernicharo, C. Kahane, M. Guelin, and J. Gomez-Gonzalez, "Tentative detection of $\mathrm{CH}_{3} \mathrm{NC}$ towards SGR B2," Astron. Astrophys. 189, L1-L2 (1988).

${ }^{34}$ A. J. Remijan, J. M. Hollis, F. J. Lovas, D. F. Plusquellic, and P. R. Jewell, "Interstellar isomers: The importance of bonding energy differences," Astrophys. J. 632, 333-339 (2005).

${ }^{35}$ P. Gratier, J. Pety, V. Guzman, M. Gerin, J. R. Goicoechea, E. Roueff, and A. Faure, "The IRAM-30 m line survey of the Horsehead PDR: III. High abundance of complex (iso-)nitrile molecules in UV-illuminated gas," Astron. Astrophys. 557, A101 (2013).

${ }^{36}$ M. Meier, B. Dogan, D. Beckhaus, and C. Ruechardt, "Heats of formation and heats of isomerization of isocyanides," New J. Chem. 11, 1-6 (1987).

${ }^{37}$ R. L. Hudson and M. H. Moore, "Reactions of nitriles in ices relevant to Titan, comets, and the interstellar medium: Formation of cyanate ion, ketenimines, and isonitriles," Icarus 172, 466-478 (2004).

${ }^{38} \mathrm{Z}$. Kisiel and A. Kraśnicki, "The millimetre-wave rotational spectrum of phenylacetylene," J. Mol. Spectrosc. 262, 82-88 (2010).

${ }^{39}$ M. A. Zdanovskaia, B. J. Esselman, H. S. Lau, D. M. Bates, R. C. Woods, R. J. McMahon, and Z. Kisiel, "The 103-360 GHz rotational spectrum of benzonitrile, the first interstellar benzene derivative detected by radioastronomy," J. Mol. Spectrosc. 351, 39-48 (2018).

${ }^{40}$ B. J. Esselman, B. K. Amberger, J. D. Shutter, M. A. Daane, J. F. Stanton, R. C. Woods, and R. J. McMahon, "Rotational spectroscopy of pyridazine and its isotopologs from 235-360 GHz: Equilibrium structure and vibrational satellites," J. Chem. Phys. 139, 224304 (2013).

${ }^{41}$ B. K. Amberger, B. J. Esselman, J. F. Stanton, R. C. Woods, and R. J. McMahon, "Precise equilibrium structure determination of hydrazoic acid $\left(\mathrm{HN}_{3}\right)$ by millimeter-wave spectroscopy," J. Chem. Phys. 143, 104310 (2015).

${ }^{42}$ Z. Kisiel, L. Pszczółkowski, I. R. Medvedev, M. Winnewisser, F. C. De Lucia, and E. Herbst, "Rotational spectrum of trans-trans diethyl ether in the ground and three excited vibrational states," J. Mol. Spectrosc. 233, 231-243 (2005).

${ }^{43}$ Z. Kisiel, L. Pszczółkowski, B. J. Drouin, C. S. Brauer, S. Yu, J. C. Pearson, I. R. Medvedev, S. Fortman, and C. Neese, "Broadband rotational spectroscopy of acrylonitrile: Vibrational energies from perturbations," J. Mol. Spectrosc. 280, 134-144 (2012).

${ }^{44}$ H. M. Pickett, "The fitting and prediction of vibration-rotation spectra with spin interactions," J. Mol. Spectrosc. 148, 371-377 (1991).

${ }^{45}$ See http://info.ifpan.edu.pl/ kisiel/prospe.htm for PROSPE-Programs for ROtational SPEctroscopy. 
${ }^{46}$ M. J. Frisch, G. W. Trucks, H. B. Schlegel, G. E. Scuseria, M. A. Robb, J. R. Cheeseman, G. Scalmani, V. Barone, G. A. Petersson, H. Nakatsuji, X. Li, M. Caricato, A. V. Marenich, J. Bloino, B. G. Janesko, R. Gomperts, B. Mennucci, H. P. Hratchian, J. V. Ortiz, A. F. Izmaylov, J. L. Sonnenberg, D. WilliamsYoung, F. Ding, F. Lipparini, F. Egidi, J. Goings, B. Peng, A. Petrone, T. Henderson, D. Ranasinghe, V. G. Zakrzewski, J. Gao, N. Rega, G. Zheng, W. Liang, M. Hada, M. Ehara, K. Toyota, R. Fukuda, J. Hasegawa, M. Ishida, T. Nakajima, Y. Honda, O. Kitao, H. Nakai, T. Vreven, K. Throssell, J. A. Montgomery, Jr., J. E. Peralta, F. Ogliaro, M. J. Bearpark, J. J. Heyd, E. N. Brothers, K. N. Kudin, V. N. Staroverov, T. A. Keith, R. Kobayashi, J. Normand, K. Raghavachari, A. P. Rendell, J. C. Burant, S. S. Iyengar, J. Tomasi, M. Cossi, J. M. Millam, M. Klene, C. Adamo, R. Cammi, J. W. Ochterski, R. L. Martin, K. Morokuma, O. Farkas, J. B. Foresman, and D. J. Fox, Gaussian 16, Revision B.01, Gaussian, Inc., Wallingford, CT, 2016.

${ }^{47}$ D. Leifert, D. G. Artiukhin, J. Neugebauer, A. Galstyan, C. A. Strassert, and A. Studer, "Radical perfluoroalkylation-Easy access to 2-perfluoroalkylindol-3imines via electron catalysis," Chem. Commun. 52, 5997-6000 (2016).

${ }^{48}$ W. P. Weber, G. W. Gokel, and I. K. Ugi, "Phase transfer catalysis in the Hofmann carbylamine reaction," Angew. Chem., Int. Ed. 11, 530-531 (1972).

${ }^{49}$ D. Knol, N. J. Koole, and M. J. A. de Bie, "A ${ }^{13} \mathrm{C}$ NMR investigation of some tetrakisisocyanocopper(I) tetrafluoroborate complexes: Chemical shift of, and coupling to, the isocyano carbon," Org. Magn. Reson. 8, 213-218 (1976).

${ }^{50}$ B. Kim, J. M. Beebe, Y. Jun, X. Y. Zhu, and C. D. Frisbie, "Correlation between HOMO alignment and contact resistance in molecular junctions: Aromatic thiols versus aromatic isocyanides," J. Am. Chem. Soc. 128, 4970-4971 (2006).
${ }^{51}$ J. A. Green and P. T. Hoffmann, "The structure of isonitriles," in Isonitrile Chemistry, edited by I. Ugi (Academic Press, New York, 1971), pp. 1-7.

${ }^{52} \mathrm{~J}$. K. G. Watson, "Aspects of quartic and sextic centrifugal effects on rotational energy levels," in Vibrational Structure and Spectra, edited by J. R. Durig (Elsevier, Amsterdam, 1977), Vol. 6, pp. 1-89.

${ }^{53}$ For benzonitrile, combining the large number of hyperfine-resolved transitions reported in the literature with the very large number of new, hyperfine-unresolved transitions determined in our recent millimeter-wave study afforded refined values of the quadrupole coupling constants in the least-squares fit of the data. By contrast, far fewer hyperfine-resolved transitions have been reported in the literature for phenyl isocyanide; including these values with our new hyperfineunresolved data did not result in an improvement in the values of the quadrupole coupling constants for phenyl isocyanide in the current analysis.

${ }^{54}$ M. Piccardo, E. Penocchio, C. Puzzarini, M. Biczysko, and V. Barone, "Semiexperimental equilibrium structure determinations by employing B3LYP/SNSD anharmonic force fields: Validation and application to semirigid organic molecules," J. Phys. Chem. A 119, 2058-2082 (2015).

${ }^{55}$ M. H. Palmer, R. Wugt Larsen, and F. Hegelund, "Comparison of theoretical and experimental studies of infrared and microwave spectral data for 5- and 6-membered ring heterocycles: The rotation constants, centrifugal distortion and vibration rotation constants," J. Mol. Spectrosc. 252, 60-71 (2008).

${ }^{56}$ W. Gordy and R. Cook, Microwave Molecular Spectra, 3rd ed. (Wiley, New York, 1984).

${ }^{57}$ Z. Kisiel, E. B. Jaworska, R. A. H. Butler, D. T. Petkie, P. Helminger, I. R. Medvedev, and F. C. De Lucia, "The rotational spectrum of chlorine nitrate $\left(\mathrm{ClONO}_{2}\right)$ in the four lowest $n v_{9}$ polyads," J. Mol. Spectrosc. 254, 78-86 (2009). 